\title{
Graphical condensation, overlapping Pfaffians and superpositions of matchings
}

\author{
Markus Fulmek * \\ Submitted: Dec 10, 2009; Accepted: May 25, 2010; Published: Jun 7, 2010 \\ Mathematics Subject Classification: 05C70 05A19 05E99
}

\begin{abstract}
The purpose of this paper is to exhibit clearly how the "graphical condensation" identities of Kuo, Yan, Yeh and Zhang follow from classical Pfaffian identities by the Kasteleyn-Percus method for the enumeration of matchings. Knuth termed the relevant identities "overlapping Pfaffian" identities and the key concept of proof "superpositions of matchings". In our uniform presentation of the material, we also give an apparently unpublished general "overlapping Pfaffian" identity of Krattenthaler.
\end{abstract}

\section{Introduction}

In the last 7 years, several authors $[11,12,16,22,23]$ came up with identities related to the enumeration of matchings in planar graphs, together with a beautiful method of proof, which they termed graphical condensation.

In this paper, we show that these identities are special cases of certain Pfaffian identities (in the simplest case Tanner's identity [19]), by simply applying the Kasteleyn-Percus method [7, 15]. These identities involve products of Pfaffians, for which Knuth [9] coined the term overlapping Pfaffians. Overlapping Pfaffians were further investigated by Hamel [6].

Knuth gave a very clear and concise exposition not only of the results, but also of the main idea of proof, which he termed superposition of matchings.

Tanner's identity dates back to the 19th century — and so does the basic idea of superposition of matchings, which was used for a proof of Cayley's Theorem [1] by Veltmann

\footnotetext{
*Research supported by the National Research Network "Analytic Combinatorics and Probabilistic Number Theory", funded by the Austrian Science Foundation.
} 
in 1871 [20] and independently by Mertens in 1877 [13] (as was already pointed out by Knuth [9]). Basically the same proof of Cayley's Theorem was presented by Stembridge [18], who gave a very elegant "graphical" description of Pfaffians.

The purpose of this paper is to exhibit clearly how "graphical condensation" is connected to "overlapping Pfaffian" identities. This is achieved by

- using Stembridge's description of Pfaffians to give a simple, uniform presentation of the underlying idea of "superposition of matchings", accompanied by many graphical illustrations (which should demonstrate ad oculos the beauty of this idea),

- using this idea to give uniform proofs for several known "overlapping Pfaffian" identities and a general "overlapping Pfaffian" identity, which to the best of our knowledge is due to Krattenthaler [10] and was not published before,

- and (last but not least) making clear how the "graphical condensation" identities of Kuo [11, Theorem 2.1 and Theorem 2.3], Yan, Yeh and Zhang [23, Theorem 2.2 and Theorem 3.2] and Yan and Zhang [22, Theorem 2.2] follow immediately from the "overlapping Pfaffian" identities via the classical Kasteleyn-Percus method for the enumeration of (perfect) matchings.

This paper is organized as follows:

- Section 2 presents the basic definitions and notations used in this paper,

- Section 3 presents the concept of "superposition of matchings", using a simple instance of "graphical condensation" as an introductory example,

- Section 4 presents Stembridge's description of Pfaffians,

- Section 5 recalls the Kasteleyn-Percus method,

- Section 6 presents Tanner's classical identity and more general "overlapping Pfaffian" identities, together with "superposition of matchings"-proofs, and deduces the "graphical condensation" identities [11, Theorem 2.1 and Theorem 2.3], [23, Theorem 2.2 and Theorem 3.2] and [22, Theorem 2.2].

\section{Basic notation: Ordered sets (words), graphs and matchings}

The sets we shall consider in this paper will always be finite and ordered, whence we may view them as words of distinct letters

$$
\alpha=\left\{\alpha_{1}, \alpha_{2}, \ldots, \alpha_{n}\right\} \simeq\left(\alpha_{1}, \alpha_{2}, \ldots, \alpha_{n}\right) .
$$


When considering some subset $\gamma \subseteq \alpha$, we shall always assume that the elements (letters) of $\gamma$ appear in the same order as in $\alpha$, i.e.,

$$
\gamma=\left\{\alpha_{i_{1}}, \alpha_{i_{2}}, \ldots, \alpha_{i_{k}}\right\} \simeq\left(\alpha_{i_{1}}, \alpha_{i_{2}}, \ldots, \alpha_{i_{k}}\right) \text { with } i_{1}<i_{2}<\cdots<i_{k} .
$$

We choose this somewhat indecisive notation because the order of the elements (letters) is not always relevant. For instance, for graphs $G$ we shall employ the usual (set-theoretic) terminology: $G=G(\mathrm{~V}, \mathrm{E})$ with vertex set $\mathrm{V}(G)=\mathrm{V}$ and edge set $\mathrm{E}(G)=\mathrm{E}$, the ordering of $V$ is irrelevant for typical graph-theoretic questions like "is $G$ a planar graph?".

The graphs we shall consider in this paper will always be finite and loopless (they may, however, have multiple edges). Moreover, the graphs will always be weighted, i.e., we assume a weight function $\omega: \mathrm{E}(G) \rightarrow R$, where $R$ is some integral domain. (If we are interested in mere enumeration, we may simply choose $\omega \equiv 1$.)

The weight $\omega(U)$ of some subset of edges $U \subseteq \mathrm{E}(G)$ is defined as

$$
\omega(U):=\prod_{e \in U} \omega(e)
$$

The total weight (or generating function) of some family $\mathcal{F}$ of subsets of $\mathrm{E}(G)$ is defined as

$$
\omega(\mathcal{F}):=\sum_{U \in \mathcal{F}} \omega(U)
$$

For some subset $S \subseteq \mathrm{V}(G)$, we denote by $[G-S]$ the subgraph of $G$ induced by the vertex set $(\mathrm{V}(G) \backslash S)$.

A matching in $G$ is a subset $\mu \subseteq \mathrm{E}(G)$ of edges such that

- no two edges in $\mu$ have a vertex in common,

- and every vertex in $\mathrm{V}(G)$ is incident with precisely one edge in $\mu$.

(This concept often is called a perfect matching). Note that a matching $\mu$ may be viewed as a partition of $\mathrm{V}(G)$ into blocks (subsets) of cardinality 2 (every $e \in \mu$ forms a block).

\section{Kuo's Proposition and superposition of matchings}

Denote the family of all matchings of $G$ by $\mathcal{M}_{G}$, and denote the total weight of all matchings of $G$ by $M_{G}:=\omega\left(\mathcal{M}_{G}\right)$.

According to Kuo [11], the following proposition is a generalization of results of Propp [16, section 6] and Kuo [12], and was first proved combinatorially by Yan, Yeh and Zhang [23]: 
Proposition 1. Let $G$ be a planar graph with four vertices $a, b, c$ and $d$ that appear in that cyclic order on the boundary of a face of $G$. Then

$$
M_{G} M_{[G-\{a, b, c, d\}]}+M_{[G-\{a, c\}]} M_{[G-\{b, d\}]}=M_{[G-\{a, b\}]} M_{[G-\{c, d\}]}+M_{[G-\{a, d\}]} M_{[G-\{b, c\}\}} .
$$

As we will see, this statement is a direct consequence of Tanner's [19] identity (see [9, Equation (1.0)]) and the Kasteleyn-Percus method [8], but we shall use it here as a simple example to introduce the concept of superposition of matchings, as the straightforward combinatorial intepretation of the products involved in equations like (1) was termed by Knuth [9].

\subsection{Superpositions of matchings}

Consider a simple graph $G$ and two disjoint (but otherwise arbitrary) subsets of vertices $\mathbf{b} \subseteq \mathrm{V}(G)$ and $\mathbf{r} \subseteq \mathrm{V}(G)$. Call $\mathbf{b}$ the blue vertices, $\mathbf{r}$ the red vertices, $\mathbf{c}:=\mathbf{r} \cup \mathbf{b}$ the coloured vertices and the remaining $\mathbf{w}:=\mathrm{V}(G) \backslash \mathbf{c}$ the white vertices. Now consider the bicoloured graph $B=G_{\mathrm{r} \mid \mathbf{b}}$

- with vertex set $\mathrm{V}(B):=\mathrm{V}(G)$,

- and with edge set $\mathrm{E}(B)$ equal to the disjoint union of

- the edges of $\mathrm{E}([G-\mathbf{b}])$, which are coloured red,

- and the edges of $\mathrm{E}([G-\mathrm{r}])$, which are coloured blue.

Here, "disjoint union" should be understood in the sense that $\mathrm{E}([G-\mathbf{b}])$ and $\mathrm{E}([G-\mathbf{r}])$ are subsets of two different "copies" of $\mathrm{E}(G)$, respectively. This concept will appear frequently in the following: assume that we have two copies of some set $M$. We may imagine these copies to have different colours, red and blue, and denote them accordingly by $M_{r}$ and $M_{b}$, respectively. Then "by definition" subsets $M_{r}^{\prime} \subseteq M_{r}$ and $M_{b}^{\prime} \subseteq M_{b}$ are disjoint: every element in $M_{r}^{\prime} \cap M_{b}^{\prime}$ (in the ordinary sense, as subsets of $M$ ) appears twice (as red copy and as blue copy) in $M_{r}^{\prime} \cup M_{b}^{\prime}$. Introducing the notation $X \cup \dot{\cup} Y$ as a shortcut for disjoint union, i.e, for " $X \cup Y$, where $X \cap Y=\emptyset$ ", we can write:

$$
\mathrm{E}(B)=\mathrm{E}([G-\mathbf{b}]) \dot{\cup} \mathrm{E}([G-\mathbf{r}]) .
$$

Note that in $B=G_{\mathbf{r} \mid \mathbf{b}}$

- all edges incident with blue vertices (i.e., with vertices in $\mathbf{b}$ ) are blue,

- all edges incident with red vertices (i.e., with vertices in r) are red,

- and all edges in $\mathrm{E}([G-\mathbf{c}])$ appear as double edges in $\mathrm{E}(B)$; one coloured red and the other coloured blue. 
Figure 1: Illustration: A graph $G$ with two disjoint subsets of vertices $\mathbf{r}$ and $\mathbf{b}$ (shown in the left picture) gives rise to the bicoloured graph $G_{\mathbf{r} \mid \mathbf{b}}$ (shown in the right picture; blue edges are shown as dashed lines).
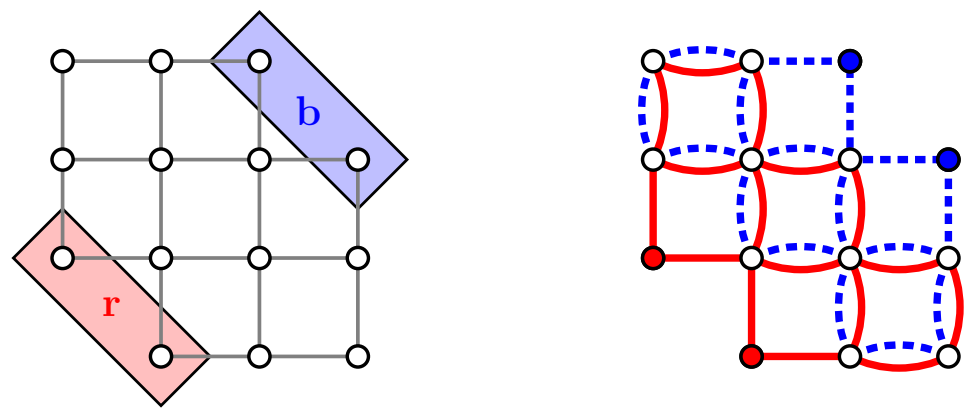

See Figure 1 for an illustration.

The weight function $\omega$ on the edges of graph $B=G_{\mathrm{r} \mid \mathbf{b}}$ is assumed to be inherited from graph $G: \omega(e)$ in $B$ equals $\omega(e)$ in $G$ (irrespective of the colour of $e$ in $B$ ).

Observation 1 (superposition of matchings). Define the weight $\omega(X, Y)$ of a pair $(X, Y)$ of subsets of edges as

$$
\omega(X, Y):=\omega(X) \cdot \omega(Y) .
$$

Then $M_{[G-\mathbf{b}]} M_{[G-\mathrm{r}]}$ clearly equals the total weight of $\mathcal{M}_{[G-\mathbf{b}]} \times \mathcal{M}_{[G-\mathbf{r}]}$, since the typical summand in $M_{[G-\mathbf{b}]} M_{[G-\mathbf{r}]}$ is $\omega\left(\mu_{r}\right) \cdot \omega\left(\mu_{b}\right)=\omega\left(\mu_{r}, \mu_{b}\right)$, where $\left(\mu_{r}, \mu_{b}\right)$ is of some pair of matchings $\left(\mu_{r}, \mu_{b}\right) \in \mathcal{M}_{[G-\mathbf{b}]} \times \mathcal{M}_{[G-\mathrm{r}]}$. Such pair of matchings can be viewed as the disjoint union $\mu_{r} \dot{\cup} \mu_{b} \subseteq \mathrm{E}(B)$ in the bicoloured graph $B$, where $\mu_{r}$ is a subset of the red edges, and $\mu_{b}$ is a subset of the blue edges. We call any subset in $\mathrm{E}(B)$ which arises from a pair of matchings in this way a superposition of matchings, and we denote by $\mathcal{S}_{B}$ the family of superpositions of matchings of $B$. So there is a weight preserving bijection

$$
\mathcal{M}_{[G-\mathrm{b}]} \times \mathcal{M}_{[G-\mathrm{r}]} \leftrightarrow \mathcal{S}_{B}
$$

Observation 2 (nonintersecting bicoloured paths/cycles). It is obvious that some subset $S \subseteq \mathrm{E}(B)$ of edges of the bicoloured graph $B$ is a superposition of matchings if and only if

- every blue vertex $v$ (i.e., $v \in \mathbf{b}$ ) is incident with precisely one blue edge from $S$,

- every red vertex $v$ (i.e., $v \in \mathrm{r}$ ) is incident with precisely one red edge from $S$,

- every white vertex $v$ (i.e., $v \in \mathbf{w}$ ) is incident with precisely one blue edge and precisely one red edge from $S$.

Stated otherwise: A superposition of matchings in $B$ may be viewed as a family of paths and cycles, 
- such that every vertex of $B$ belongs to precisely one path or cycle (i.e., the paths/cycles are nonintersecting: no two different cycles/paths have a vertex in common),

- such that edges of each cycle/path alternate in colour along the cycle/path (therefore, we call them bicoloured: Note that a bicoloured cycle must have even length),

- such that precisely the end vertices of paths are coloured (i.e., red or blue), and all other vertices are white.

Note that a bicoloured cycle of length $>2$ in the bicoloured graph $B=G_{\mathbf{r} \mid \mathbf{b}}$ corresponds to a cycle in $G$, while a bicoloured cycle of length 2 in $B$ corresponds to a "doubled edge" in $G$.

Observation 3 (colour-swap along paths). For an arbitrary coloured vertex $x$ in some superposition of matchings $S$ of $\mathrm{E}(B)$, we may swap colours for all the edges in the unique path $p$ in $S$ with end vertex $x$ (see Figure 2). Without loss of generality, assume that $x$ is red. Depending on the colour of the other end vertex $y$ of $p$, this colour-swap results in a set of coloured edges $\bar{S}$, which is a superposition of matchings in

- $B^{\prime}=G_{\mathbf{r}^{\prime} \mid \mathbf{b}^{\prime}}$, where $\mathbf{r}^{\prime}:=(\mathbf{r} \backslash\{x\}) \cup\{y\}$ and $\mathbf{b}^{\prime}:=(\mathbf{b} \backslash\{y\}) \cup\{x\}$, if $y$ is blue (i.e., of the opposite colour as $x$; the length of the path $p$ is even in this case - this case is illustrated in Figure 2),

- $B^{\prime \prime}=G_{\mathbf{r}^{\prime \prime} \mid \mathbf{b}^{\prime \prime}}$, where $\mathbf{r}^{\prime \prime}:=(\mathbf{r} \backslash\{x, y\})$ and $\mathbf{b}^{\prime \prime}:=\mathbf{b} \cup\{x, y\}$, if $y$ is red (i.e., of the same colour as $x$; the length of the path $p$ is odd in this case).

Clearly, this operation of swapping colours defines a weight preserving injection

$$
\chi_{x}: \mathcal{S}_{B} \rightarrow\left(\mathcal{S}_{B^{\prime}} \cup \mathcal{S}_{B^{\prime \prime}}\right)
$$

(which, viewed as mapping onto its image, is an involution: $\chi_{x}=\chi_{x}^{-1}$ ). So $\chi_{x}$ together with the bijection (2) gives a weight preserving injection

$$
\mathcal{M}_{[G-\mathbf{b}]} \times \mathcal{M}_{[G-\mathbf{r}]} \rightarrow\left(\bigcup_{B^{\prime}} \mathcal{M}_{\left[G-\mathbf{b}^{\prime}\right]} \times \mathcal{M}_{\left[G-\mathbf{r}^{\prime}\right]}\right) \cup\left(\bigcup_{B^{\prime \prime}} \mathcal{M}_{\left[G-\mathbf{b}^{\prime \prime}\right]} \times \mathcal{M}_{\left[G-\mathbf{r}^{\prime \prime}\right]}\right)
$$

where the unions are over all bicoloured graphs $B^{\prime}$ and $B^{\prime \prime}$ that arise from the recolouring of the path $p$, as described above.

\subsection{The "graphical condensation method"}

Now we apply the reasoning outlined in Observations 1, 2 and 3 for the proof of Proposition 1 (basically the same proof is presented in [11]): 
Figure 2: Illustration: Take graph $G$ of Figure 1 and consider a matching in $[G-\mathrm{r}]$ $(\mathrm{r}=\{x, t\})$, whose edges are colored blue (shown as dashed lines), and a matching in $[G-\mathbf{b}](\mathbf{b}=\{y, z\})$, whose edges are colored red. This superposition of matchings

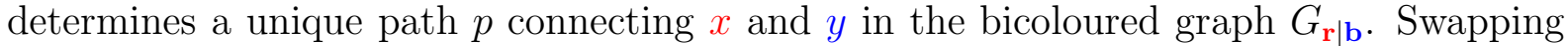
the colours of the edges of $p$ determines uniquely a matching in $\left[G-\mathbf{r}^{\prime}\right]\left(\mathbf{r}^{\prime}=\{y, t\}\right)$ and a matching in $\left[G-\mathbf{b}^{\prime}\right]\left(\mathbf{b}^{\prime}=\{x, z\}\right)$.

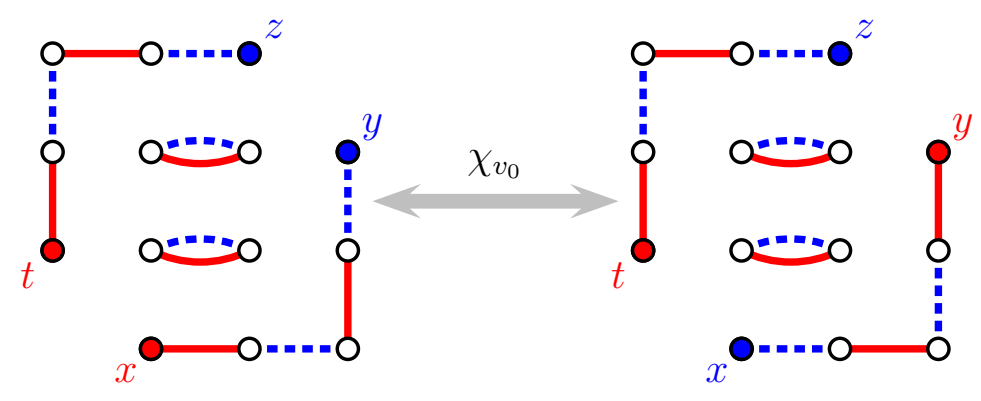

Proof of Proposition 1. Clearly, for all the superpositions of matchings (see Observation 1) involved in (1), the set $\mathbf{c}$ of coloured vertices in the associated bicoloured graphs is $\{a, b, c, d\}$. In any superposition of matchings, there are two nonintersecting paths (see Observation 2) with end vertices in $\{a, b, c, d\}$. Since $G$ is planar and the vertices $a, b, c$ and $d$ appear in this cyclic order in the boundary of a face $F$ of $G$, the path starting in vertex $a$ cannot end in vertex $c$ (otherwise it would intersect the path connecting $b$ and $d$; see Figure 3 for an illustration).

So consider the bicoloured graphs

- $B_{1}:=G_{\mathrm{r}_{1} \mid \mathbf{b}_{1}}$ with $\mathbf{r}_{1}:=\{a, b, c, d\}, \mathbf{b}_{1}=\emptyset$,

- and $B_{2}:=G_{\mathrm{r}_{2} \mid \mathbf{b}_{2}}$ with $\mathbf{r}_{2}:=\{a, c\}, \mathbf{b}_{2}=\{b, d\}$.

Observe that

$$
\begin{aligned}
& M_{G} M_{\left[G-\mathrm{r}_{1}\right]}+M_{\left[G-\mathbf{b}_{2}\right]} M_{\left[G-\mathrm{r}_{2}\right]}= \\
& \omega\left(\left(\mathcal{M}_{\left[G-\mathbf{b}_{1}\right]} \times \mathcal{M}_{\left[G-\mathrm{r}_{\mathbf{1}}\right]}\right) \dot{\cup}\left(\mathcal{M}_{\left[G-\mathbf{b}_{2}\right]} \times \mathcal{M}_{\left[G-\mathrm{r}_{2}\right]}\right)\right)=\omega\left(\mathcal{S}_{B_{1}} \dot{\cup} \mathcal{S}_{B_{2}}\right) .
\end{aligned}
$$

Note that for any superposition of matchings, the other end-vertex of the bicoloured path starting at $a$ necessarily has

- the same colour as $a$ in $B_{1}$ (i.e., red),

- the other colour as $a$ in $B_{2}$ (i.e., blue). 
Figure 3: A simple planar graph $G$ with vertices $a, b, c$ and $d$ appearing in this order in the boundary of face $F$.

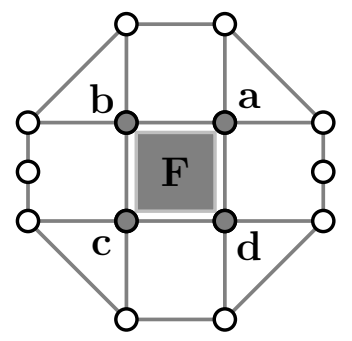

(See Figure 4.) So consider the bicoloured graphs

- $B_{1}^{\prime}:=G_{\mathbf{r}_{1}^{\prime} \mid \mathbf{b}_{1}^{\prime}}$ with $\mathbf{r}_{1}^{\prime}:=\{b, c\}, \mathbf{b}_{1}^{\prime}=\{a, d\}$,

- and $B_{2}^{\prime}:=G_{\mathbf{r}_{2}^{\prime}} \mid \mathbf{b}_{2}^{\prime}$ with $\mathbf{r}_{2}^{\prime}:=\{c, d\}, \mathbf{b}_{2}^{\prime}=\{a, b\}$.

It is easy to see that the operation $\chi_{a}$ of swapping colours of edges along the path starting at vertex $a$ (see Observation 3) defines a weight preserving involution

$$
\chi_{a}: \mathcal{S}_{B_{1}} \cup \dot{\mathcal{S}_{B_{2}}} \leftrightarrow \mathcal{S}_{B_{1}^{\prime}} \dot{\cup} \mathcal{S}_{B_{2}^{\prime}}
$$

and thus gives a weight preserving involution

$$
\begin{aligned}
\left(\mathcal{M}_{G} \times \mathcal{M}_{\left[G-\mathrm{r}_{1}\right]}\right) \dot{U}\left(\mathcal{M}_{\left[G-\mathrm{b}_{2}\right]} \times \mathcal{M}_{\left[G-\mathrm{r}_{2}\right]}\right) \leftrightarrow & \\
& \left(\mathcal{M}_{\left[G-\mathrm{b}_{1}^{\prime}\right]} \times \mathcal{M}_{\left[G-\mathrm{r}_{1}^{\prime}\right]}\right) \dot{\cup}\left(\mathcal{M}_{\left[G-\mathrm{b}_{2}^{\prime}\right]} \times \mathcal{M}_{\left[G-\mathrm{r}_{2}^{\prime}\right]}\right) .
\end{aligned}
$$

(See Figure 4 for an illustration.)

This bijective proof certainly is very satisfactory. But since there is a well-known powerful method for enumerating perfect matchings in planar graphs, namely the Kasteleyn-Percus method (see $[7,8,15]$ ) which involves Pfaffians, the question arises whether Proposition 1 (or the bijective method of proof) gives additional insight or provides a new perspective.

\section{Pfaffians}

The name Pfaffian was introduced by Cayley [2] (see [9, page 10f] for a concise historical survey). Here, we follow closely Stembridge's exposition [18]: 
Figure 4: Take the planar graph $G$ from Figure 3 and consider superpositions of matchings in the bicoloured graphs $B_{1}, B_{2}, B_{1}^{\prime}$ and $B_{2}^{\prime}$ from the proof of Proposition 1: The pictures in the upper half show two superpositions of matchings (the edges belonging to the matchings are drawn as thick lines, the blue edges appear as dashed lines) in each of the two bicoloured graphs $B_{1}$ and $B_{2}$, which are mapped to superpositions of matchings in $B_{1}^{\prime}$ and $B_{2}^{\prime}$, respectively, by the operation $\chi_{a}$ (i.e., by swapping colours of edges in the unique bicoloured path with end vertex $a$ ). The mapping given by $\chi_{a}$ is indicated by arrows.

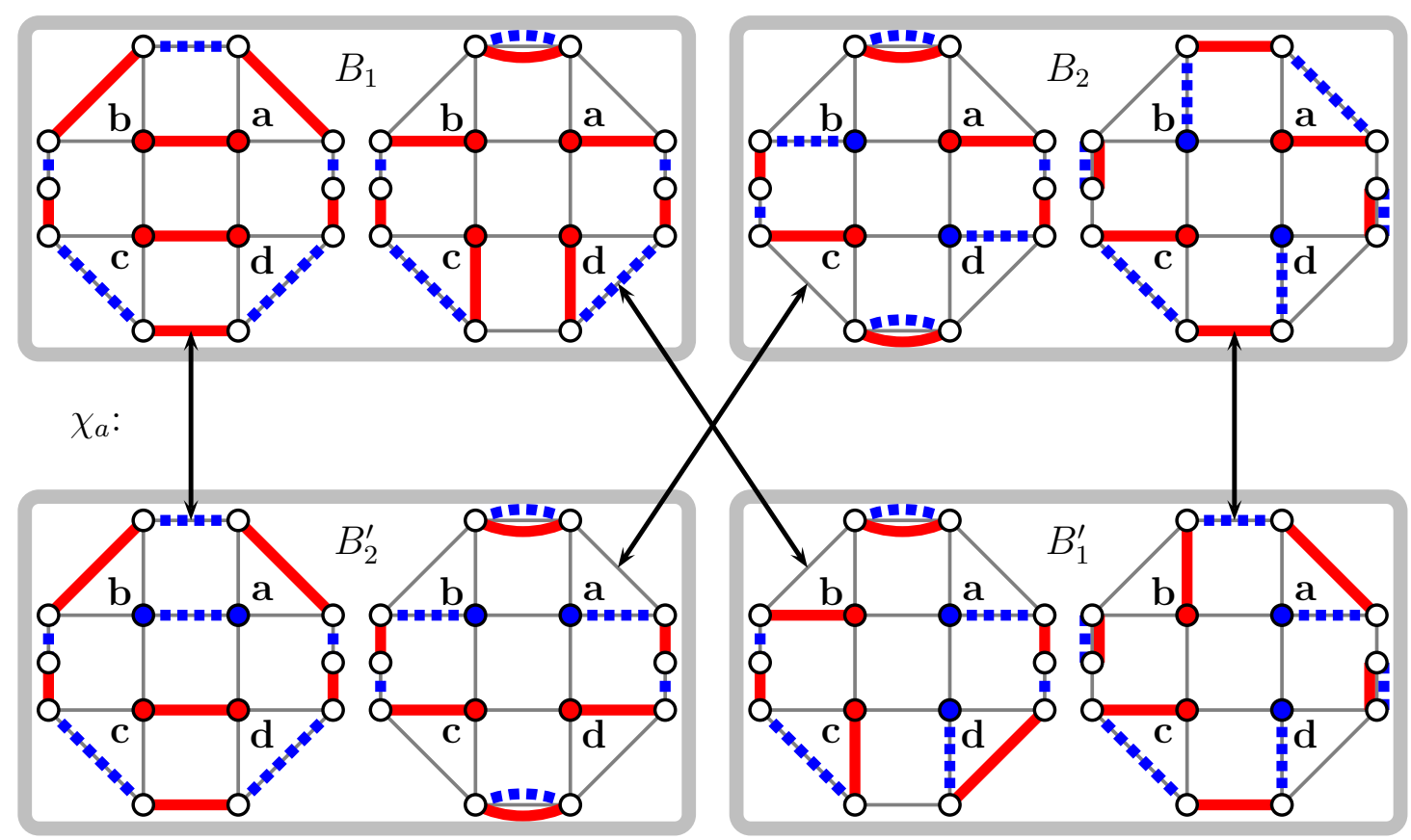


Definition 1. Consider the complete graph $K_{V}$ on the (ordered) set of vertices $V=$ $\left(v_{1}, \ldots v_{n}\right)$, with weight function $\omega: \mathrm{E}\left(K_{V}\right) \rightarrow R$. Draw this graph in the upper halfplane in the following way:

- Vertex $v_{i}$ is represented by the point $(i, 0)$,

- edge $\left\{v_{i}, v_{j}\right\}$ is represented by the half-circle with center $\left(\frac{i+j}{2}, 0\right)$ and radius $\frac{|j-i|}{2}$ in the upper half-plane.

(See the left picture in Figure 5).

Consider some matching $\mu=\left\{\left\{v_{i_{1}}, v_{j_{1}}\right\}, \ldots,\left\{v_{i_{m}}, v_{j_{m}}\right\}\right\}$ in $K_{V}$. Clearly, if such $\mu$ exists, then $n=2 m$ must be even. By convention, we assume $i_{k}<j_{k}$ for $k=1, \ldots, m$. A crossing of $\mu$ corresponds to a crossing of edges in the specific drawing just described, or more formally: A crossing of $\mu$ is a pair of edges $\left(\left\{v_{i_{k}}, v_{j_{k}}\right\},\left\{v_{i_{l}}, v_{j_{l}}\right\}\right)$ of $\mu$ such that

$$
i_{k}<i_{l}<j_{k}<j_{l} \text {. }
$$

Then the sign of $\mu$ is defined as

$$
\operatorname{sgn}(\mu):=(-1)^{\#(\text { crossings of } \mu)} .
$$

(See the right picture in Figure 5).

Now arrange the weights $a_{i, j}:=\omega\left(\left\{v_{i}, v_{j}\right\}\right)$ in an upper triangular array $A=\left(a_{i, j}\right)_{1 \leqslant i<j \leqslant n}$ : The Pfaffian of $A$ is defined as

$$
\operatorname{Pf}(A):=\sum_{\mu \in \mathcal{M}_{K_{V}}} \operatorname{sgn}(\mu) \omega(\mu),
$$

where the sum runs over all matchings of $K_{V}$.

Since we always view $K_{V}$ as weighted graph (with some weight function $\omega$ ), we also write $\operatorname{Pf}\left(K_{V}\right)$, or even simpler $\operatorname{Pf}(V)$, instead of $\operatorname{Pf}(A)$. We set $\operatorname{Pf}(\emptyset):=1$ by definition.

Since an upper triangular matrix $A$ determines uniquely a skew symmetric matrix $A^{\prime}$ (by letting $A_{i, j}^{\prime}=A_{i, j}$ if $j>i$ and $A_{i, j}^{\prime}=-A_{j, i}$ if $\left.j<i\right)$, we also write $\operatorname{Pf}\left(A^{\prime}\right)$ instead of $\operatorname{Pf}(A)$.

With regard to the identities for matchings we are interested in, an edge not present in graph $G$ may safely be added if it is given weight zero. Hence every simple finite weighted graph $G$ may be viewed as a subgraph (in general not an induced subgraph!) of $K_{V}$ with $\mathrm{V}\left(K_{V}\right)=\mathrm{V}(G)$, where the weight of edge $e$ in $K_{V}$ is defined to be

- $\omega(e)$ in $G$, if $e \in \mathrm{E}(G)$,

- zero, if $e \notin \mathrm{E}(G)$. 
Figure 5: Pfaffians according to Definition 1: The left picture shows $K_{4}$, drawn in the specific way described in Definition 1. The right picture shows the matching $\mu=\left\{\left\{v_{1}, v_{3}\right\},\left\{v_{2}, v_{4}\right\}\right\}$ : Since there is precisely one crossing of edges in the picture, $\operatorname{sgn}(\mu)=(-1)^{1}=-1$.
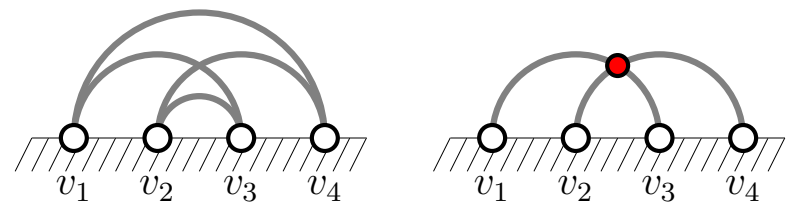

Figure 6: The contribution of edge $e=\left\{v_{i}, v_{j}\right\}$ to the sign of the matching $\pi$ amounts to $(-1)^{\#\left(\text { vertices between } v_{i} \text { and } v_{j}\right)}$ (which is the same as $(-1)^{i-j-1}$ if the vertices $\left\{v_{1}, v_{2}, \ldots, v_{2 n}\right\}$ appear in ascending order).

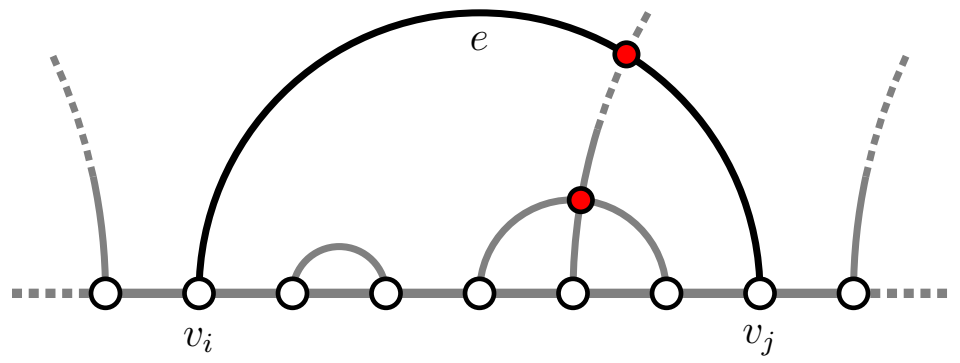

Keeping that in mind, we also write $\operatorname{Pf}(G)$ (or $\operatorname{Pf}(V)$, again) instead of $\operatorname{Pf}\left(K_{V}\right)$.

The following simple observation is central for many of the following arguments.

Observation 4 (contribution of a single edge to the sign of some matching). Let $V=$ $\left(v_{1}, \ldots, v_{2 n}\right)$. Removing an edge $e=\left\{v_{i}, v_{j}\right\}, i<j$, together with the vertices $v_{i}$ and $v_{j}$, from some matching $\mu$ of $K_{V}$ gives a matching $\bar{\mu}$ of the complete graph on the remaining vertices $\left(v_{1}, v_{2}, \ldots, v_{2 n}\right) \backslash\left\{v_{i}, v_{j}\right\}$, and the change in sign from $\mu$ to $\bar{\mu}$ is determined by the parity of the number of vertices lying between $v_{i}$ and $v_{j}$ (see Figure 6). By the ordering of the vertices, \# (vertices between $v_{i}$ and $\left.v_{j}\right)=j-i-1$, whence we have:

$$
\operatorname{sgn}(\mu)=\operatorname{sgn}(\bar{\mu}) \cdot(-1)^{j-i-1} .
$$




\subsection{Cayley's Theorem and the long history of superposition of matchings}

The following Theorem of Cayley [1] is well-known. Stembridge presented a beautiful proof (see [18, Proposition 2.2]) which was based on superposition of matchings. Basically the same proof was already found in the 19th century. We cite from [9]:

An elegant graph-theoretic proof of Cayley's theorem... was found by Veltmann in 1871 [20] and independently by Mertens in 1877 [13]. Their proof anticipated 20th-century studies on the superposition of two matchings, and the ideas have frequently been rediscovered.

Theorem 1 (Cayley). Given an upper triangular array $A=\left(a_{i, j}\right)_{1 \leqslant i<j \leqslant n}$, extend it to a skew symmetric matrix $A^{\prime}=\left(a_{i, j}^{\prime}\right)_{1 \leqslant i, j \leqslant n}$ by setting

$$
a_{i, j}^{\prime}= \begin{cases}a_{i, j} & \text { if } i<j, \\ -a_{i, j} & \text { if } i>j, \\ 0 & \text { if } i=j .\end{cases}
$$

Then we have:

$$
(\operatorname{Pf}(A))^{2}=\operatorname{det}\left(A^{\prime}\right)
$$

Cayley's Theorem is well-known, but we give the bijective proof here for two reasons:

- First, it is another beautiful application of the idea of superposition of matchings,

- and second, we need an argument from this proof for the presentation of the Kasteleyn-Percus method (in the next section).

Proof. By the definition of the determinant, we may view $\operatorname{det}\left(A^{\prime}\right)$ as the generating function of the symmetric group $\mathfrak{S}_{n}$

$$
\operatorname{det}\left(A^{\prime}\right)=\sum_{\pi \in \mathfrak{S}_{n}} \operatorname{sgn}(\pi) \omega(\pi),
$$

where the weight $\omega(\pi)$ of a permutation $\pi \in \mathfrak{S}_{n}$ is given as

$$
\omega(\pi):=\prod_{i=1}^{n} a_{i, \pi(i)}^{\prime} .
$$

The proof proceeds in two steps: 
Step 1: Denote by $\mathfrak{S}_{n}^{0}$ the set of permutations $\pi \in \mathfrak{S}_{n}$ where the cycle decomposition of $\pi$ does not contain a cycle of odd length. Then we claim:

$$
\operatorname{det}\left(A^{\prime}\right)=\sum_{\pi \in \mathfrak{S}_{n}^{0}} \operatorname{sgn}(\pi) \omega(\pi)
$$

To prove this, we define a weight-preserving and sign-reversing involution on the set of all permutations $\pi \in\left(\mathfrak{S}_{n} \backslash \mathfrak{S}_{n}^{0}\right)$ which $d o$ contain a cycle of odd length: of all odd-length cycles in $\pi$, choose the one which contains the smallest element $i$,

$$
\kappa_{1}=\left(i, \pi(i), \pi^{2}(i), \ldots, \pi^{2 m}(i)\right),
$$

and replace it by its inverse

$$
\kappa_{1}^{-1}=\left(\pi^{2 m}(i), \pi^{2 m-1}(i), \ldots, \pi(i), i\right) .
$$

This operation obviously is an involution:

$$
\pi=\underbrace{\left(\kappa_{1}, \kappa_{2}, \ldots\right)}_{\text {cycle decomposition }} \leftrightarrow \pi^{\prime}=\underbrace{\left(\kappa_{1}^{-1}, \kappa_{2}, \ldots\right)}_{\text {cycle decomposition }} .
$$

Since $\omega\left(\pi^{\prime}\right)=-\omega(\pi)$ and $\operatorname{sgn}\left(\pi^{\prime}\right)=\operatorname{sgn}(\pi)$, this involution is weight-preserving and signreversing. So the terms corresponding to $\left(\mathfrak{S}_{n} \backslash \mathfrak{S}_{n}^{0}\right)$ in the right-hand side of $(7)$ sum up to zero, which proves (8).

Step 2: We shall construct a weight- and sign-preserving bijection between the terms

- $\operatorname{sgn}(\pi) \omega(\pi)$ corresponding to the determinant as given in (8) (i.e., $\pi \in \mathfrak{S}_{n}^{0}$ )

- and $\operatorname{sgn}(\mu) \omega(\mu) \operatorname{sgn}(\nu) \omega(\nu)$ corresponding to the square of the Pfaffian in $(6)$.

To this end, consider the unique presentation of the cycle decomposition of $\pi$, i.e.

$$
\pi=\left(i_{1}, \pi\left(i_{1}\right), \pi^{2}\left(i_{1}\right), \ldots\right)\left(i_{2}, \pi\left(i_{2}\right), \pi^{2}\left(i_{2}\right), \ldots\right) \cdots\left(i_{m}, \pi\left(i_{m}\right), \pi^{2}\left(i_{m}\right), \ldots\right),
$$

where

- $i_{k}$ is the smallest element in its cycle,

- and $i_{1}<i_{2}<\cdots<i_{m}$.

Visualize $\pi$ as directed graph as follows: represent

$$
i_{1}, \pi\left(i_{1}\right), \ldots, i_{2}, \pi\left(i_{2}\right), \ldots
$$

in this order (i.e., in the order in which the elements appear in (9)) by vertices

$$
(1,0),(2,0), \ldots,(n, 0)
$$


in the plane. Call $(1,0),(3,0), \ldots$ the odd vertices, and call $(2,0),(4,0), \ldots$ the even vertices. Note that $\pi$ maps elements corresponding to even vertices to elements corresponding to odd vertices, and vice versa. If some element $i$ corresponds to an odd vertex $v$, then draw a blue semicircle arc in the upper halfplane from $v$ to the even vertex $w$ which corresponds to $\pi(i)$. If some element $j$ corresponds to an even vertex $s$, then draw a red semicircle arc in the lower halfplane from $s$ to the odd vertex $t$ which corresponds to $\pi(j)$. (See the left picture in Figure 7 for an illustration.)

Note that if we forget the orientation of the arcs, we simply have a superposition $(\mu, \nu)$ of a blue and a red matching. Some of the arcs are co-oriented (i.e., they point from left to right), and some are contra-oriented (i.e., they point from right to left). Define the sign of any such oriented superposition of matchings by

$$
\operatorname{sgn}(\mu, \nu):=\operatorname{sgn}(\mu) \cdot \operatorname{sgn}(\nu) \cdot(-1)^{\#(\text { contra-oriented arcs in } \mu \cup \nu)} .
$$

Observe that for the particular oriented superposition of matchings obtained by visualizing permutation $\pi$ as above, this definition gives precisely $\operatorname{sgn}(\pi)$. (Again, see the left picture in Figure 7 for an illustration.)

Furthermore, observe that with notation

$$
d(\pi):=|\{i: 1 \leqslant i \leqslant n, \pi(i)<i\}|
$$

we can rewrite the weight of $\pi$ as

$$
\omega(\pi)=\omega(\mu) \cdot \omega(\nu)(-1)^{d(\pi)} .
$$

However, the vertices in our graphical visualization of $\pi$ do not appear in their original order. Clearly, we can obtain the original ordering by interchanging neighbouring vertices $(i, 0)$ and $(i+1,0)$ whose corresponding elements appear in the wrong order, one after another, together with the arcs being attached to them: see the right picture in Figure 7 and observe that this interchanging of vertices does not change the sign as defined in (10). Note that after finishing this "sorting procedure", the number of contra-oriented arcs equals $d(\pi)$, so we have altogether

$$
\operatorname{sgn}(\pi)=\operatorname{sgn}(\mu) \cdot \operatorname{sgn}(\nu) \cdot(-1)^{d(\pi)} .
$$

Together with (11), this amounts to

$$
\operatorname{sgn}(\pi) \cdot \omega(\pi)=(\operatorname{sgn}(\mu) \cdot \omega(\mu)) \cdot(\operatorname{sgn}(\nu) \cdot \omega(\nu)),
$$

the right-hand side of which obviously corresponds to a term in $(\operatorname{Pf}(A))^{2}$.

On the other hand, every term in $(\operatorname{Pf}(A))^{2}$ corresponds to some superposition of matchings $S=(\mu, \nu)$, which consists only of bicoloured cycles. For a bicoloured cycle $C$ in $S$, identify the vertex $v_{C} \in C$ with the smallest label, and consider the unique blue edge $\left\{v_{C}, w_{C}\right\}$ in $C$. Orienting all bicoloured cycles $C$ such that this blue edge points "from $v_{C}$ to $w_{C}$ " gives an oriented superposition of matchings, from which we obtain a permutation without odd-length cycles and with the same weight and the same sign (by simply reversing the above "sorting procedure"). 
Figure 7: Illustration for Cayley's Theorem. The left picture shows a cycle $c$ of length 8 in some permutation $\pi$, whose smallest element is $i$, i.e.,

$$
c=\left(i, \pi(i), \pi^{2}(i), \ldots, \pi^{7}(i)\right),
$$

drawn as superposition of two directed matchings. Note that there is no crossing and precisely one contra-oriented arc, whence, according to (10), $c$ contributes $(-1)$ to the sign of $\pi$, as it should be for an even-length cycle. The right picture shows the effect of changing the position of two neighbouring vertices $a$ and $b$. For both matchings (red and blue; blue arcs appear as dashed lines), we have:

- the number of crossings changes by \pm 1 if $a$ and $b$ belong to different arcs,

- and if $a$ and $b$ belong to the same arc $e$, the orientation of $e$ is changed.

Since this amounts to a change in sign for the red matching and for the blue matching, the total effect is that the sign does not change.
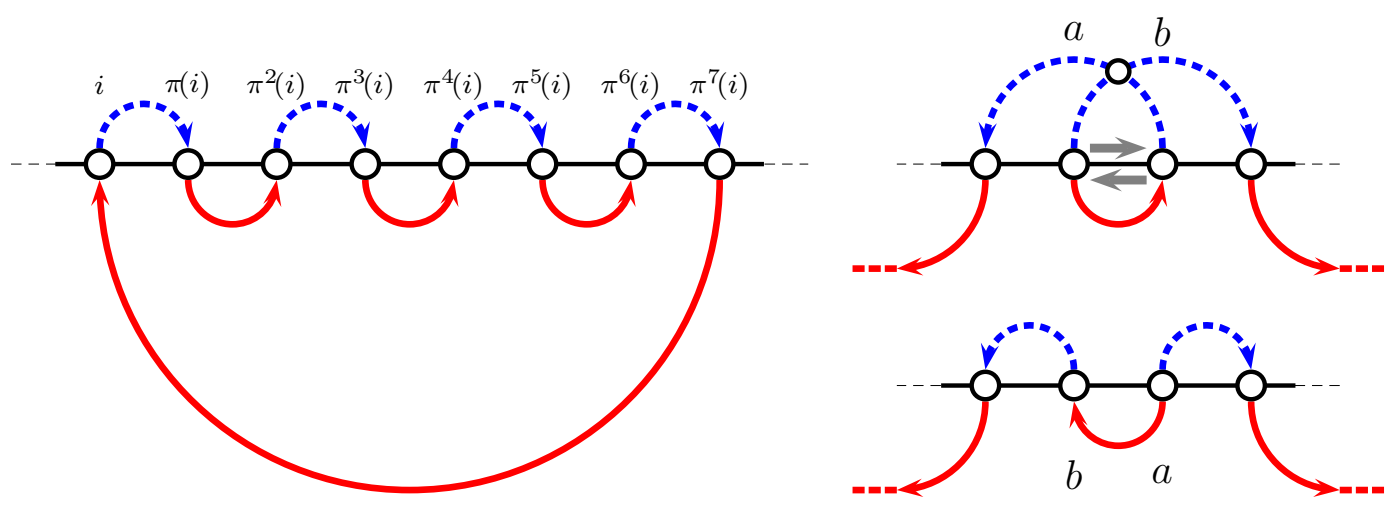


\subsection{A corollary to Cayley's Theorem}

The following Corollary is an immediate consequence of Cayley's theorem. However, we shall provide a direct "graphical" proof.

Assume that the set of vertices $V$ is partitioned into two disjoint sets $A=\left(a_{1}, \ldots, a_{m}\right)$ and $B=\left(b_{1}, \ldots, b_{n}\right)$ such that the ordered set $V$ appears as $\left(a_{1}, \ldots, a_{m}, b_{1}, \ldots, b_{n}\right)$. Denote the complete bipartite graph on $V$ (with set of edges $\left\{\left\{a_{i}, b_{j}\right\}: 1 \leqslant i \leqslant m, 1 \leqslant j \leqslant n\right\}$ ) by $K_{A: B}$. (For our purposes, we may view $K_{A: B}$ as the complete graph $K_{A \cup B}$, where $\omega\left(\left\{a_{i}, a_{j}\right\}\right)=\omega\left(\left\{b_{k}, b_{l}\right\}\right)=0$ for all $1 \leqslant i<j \leqslant m$ and for all $1 \leqslant k<l \leqslant n$.) We introduce the notation

$$
\operatorname{Pf}(A, B):=\operatorname{Pf}\left(K_{A: B}\right) .
$$

Corollary 1. Let $A=\left(a_{1}, \ldots, a_{m}\right)$ and $B=\left(b_{1}, \ldots, b_{n}\right)$ be two disjoint ordered sets. Then we have

$$
\operatorname{Pf}(A, B)= \begin{cases}(-1)^{\left(\begin{array}{c}
n \\
2
\end{array}\right)} \operatorname{det}\left(\omega\left(a_{i}, b_{j}\right)\right)_{i, j=1}^{n} & \text { if } m=n, \\
0 & \text { otherwise }\end{cases}
$$

Proof. If $m \neq n$, then there is no matching in $K_{A: B}$, and thus the Pfaffian clearly is 0 .

If $m=n$, consider the $n \times n$-matrix $M:=\left(\omega\left(\left\{a_{i}, b_{n-j+1}\right\}\right)\right)_{i, j=1}^{n}$. Note that for every permutation $\pi \in \mathfrak{S}_{n}$, the corresponding term in the expansion of $\operatorname{det}(M)$ may be viewed as the signed weight of a certain matching $\mu$ of $K_{A: B}$ (see Figure 8). Recall that $\operatorname{sgn}(\pi)=$ $(-1)^{\operatorname{inv}(\pi)}$, where $\operatorname{inv}(\pi)$ denotes the number of inversions of $\pi$, and observe that inversions of $\pi$ are in one-to-one-correspondence with crossings of $\mu$. Thus

$$
\operatorname{Pf}\left(K_{A: B}\right)=\operatorname{det}(M),
$$

and the assertion follows by reversing the order of the columns of $M$.

\subsection{A generalization of Corollary 1}

We may use Observation 4 to prove another identity involving Pfaffians. To state it conveniently, we need some further notation.

Assume that the ordered set of vertices $V$ appears as $\left(a_{1}, \ldots, a_{m}, b_{1}, \ldots, b_{n}\right)$ for disjoint sets $A=\left(a_{1}, \ldots, a_{m}\right)$ and $B=\left(b_{1}, \ldots, b_{n}\right)$. Consider the complete graph $K_{V}$ and delete (or assign weight zero to) all edges joining two vertices from $A$. Call the resulting graph the semi-bipartite graph $S_{A, B}$. (Note that every matching $\mu$ in $S_{A, B}$ constitutes an injective mapping $A \rightarrow B$.)

Let $M=\left(m_{1}, m_{2}, \ldots, m_{n}\right)$ be some ordered set. For some arbitrary subset

$$
X=\left(m_{i_{1}}, \ldots, m_{i_{k}}\right) \subseteq M,
$$


Figure 8: Illustration for Corollary 1: Consider the $4 \times 4$-matrix $M:=\left(\omega\left(\left\{a_{i}, b_{5-j}\right\}\right)\right)_{i, j=1}^{4}$ and the permutation $\pi=(2,3,4,1)$ in $\mathfrak{S}_{4}$. The left pictures shows $\pi$ as (bijective) function mapping the set $\{1,2,3,4\}$ onto itself: It is obvious that the arrows indicating the function $\pi$ constitute a matching $\mu$. The right picture shows the same matching $\mu$ drawn in the specific way of Definition 1 . Inversions of $\pi$ are in one-to-one correspondence with crossings of $\mu$, whence we see: $\operatorname{sgn}(\pi)=\operatorname{sgn}(\mu)$.
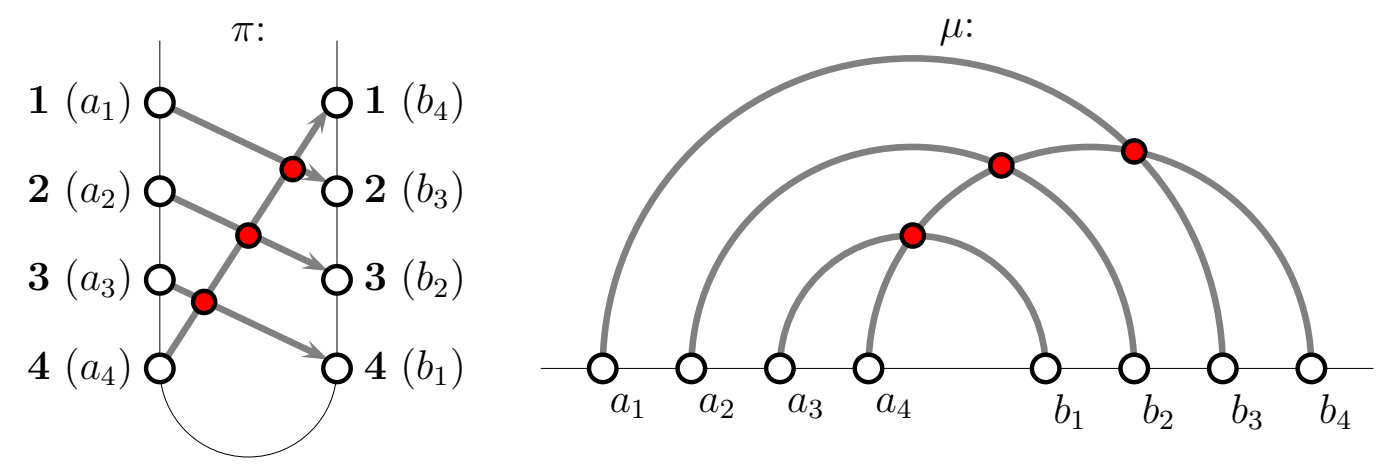

denote by $\Sigma(X \subseteq M)$ the sum of the indices $i_{j}$ of $X$ :

$$
\Sigma(X \subseteq M):=i_{1}+i_{2}+\cdots+i_{k}
$$

(Recall that we assume that subsets always "inherit" the ordering of the superset, i.e., $i_{1}<i_{2}<\cdots<i_{k}$ : we might also call $X$ a subword of $M$.)

Corollary 2. Let $V=\left(a_{1}, \ldots, a_{m}, b_{1}, \ldots, b_{n}\right), A=\left(a_{1}, \ldots, a_{m}\right)$ and $B=\left(b_{1}, \ldots, b_{n}\right)$. For every subset $Y=\left\{b_{k_{1}}, b_{k_{2}}, \ldots, b_{k_{m}}\right\} \subseteq B$, denote by $M_{Y}$ the $m \times m$-matrix

$$
M_{Y}:=\left(\omega\left(\left\{a_{i}, b_{k_{j}}\right\}\right)\right)_{i, j=1}^{m} .
$$

Then we have

$$
\operatorname{Pf}\left(S_{A, B}\right)=(-1)^{m} \sum_{\substack{Y \subseteq B \\|Y|=m}}(-1)^{\Sigma(Y \subseteq B)} \cdot \operatorname{Pf}(B \backslash Y) \cdot \operatorname{det}\left(M_{Y}\right) .
$$

Proof. For every matching $\rho$ in $S_{A, B}$, let $Y \subseteq B$ be the set of vertices which are joined with a vertex in $A$ by some edge in $\rho$. Note that $|Y|=|A|=m$ (if such matching exists), and observe that $\rho$ may be viewed as a superposition of matchings, namely

- a red matching $\mu$ in the complete bipartite graph $K_{A, Y}$

- and a blue matching $\nu$ in the complete graph $K_{B \backslash Y}$, 
where

$$
\omega(\rho)=\omega(\mu) \cdot \omega(\nu) .
$$

For an illustration, see Figure 9. Note that the crossings of $\rho$ are partitioned in

- crossings of two edges from $\mu$,

- crossings of two edges from $\nu$

- and crossings of an edge from $\mu$ with an edge from $\nu$,

whence we have

$$
\operatorname{sgn}(\rho)=(-1)^{\#(\text { crossings of an edge from } \mu \text { and an edge from } \nu)} \cdot \operatorname{sgn}(\mu) \cdot \operatorname{sgn}(\nu) .
$$

Assume that $Y=\left(b_{k_{1}}, b_{k_{2}}, \ldots, b_{k_{m}}\right)$ and observe that modulo 2 the number of crossings

- of the edge from $\mu$ which ends in $b_{k_{j}}$

- with edges from $\nu$

equals the number of vertices of $B \backslash Y$ which lie to the left of $b_{k_{j}}$, which is $k_{j}-j$. Hence we have

$$
\operatorname{sgn}(\rho) \cdot \operatorname{sgn}(\mu) \cdot \operatorname{sgn}(\nu)=(-1)^{\left(k_{1}-1\right)+\left(k_{2}-2\right)+\cdots+\left(k_{m}-m\right)}=(-1)^{\boldsymbol{\Sigma}(Y \subseteq B)-\left(\begin{array}{c}
m+1 \\
2
\end{array}\right)} .
$$

From this we obtain

$$
\operatorname{Pf}\left(S_{A, B}\right)=(-1)^{m} \sum_{\substack{Y \subset B,|Y|=m}}(-1)^{\Sigma(Y \subseteq B)} \cdot \operatorname{Pf}(B \backslash Y) \cdot(-1)^{\left(\begin{array}{c}
m \\
2
\end{array}\right)} \cdot \operatorname{Pf}(A, Y),
$$

which by Corollary 1 equals (12).

\section{Matchings and Pfaffians: The Kasteleyn-Percus method}

We now present the Kasteleyn-Percus method for the enumeration of matchings in plane graphs, following closely (and refining slightly) the exposition in [8].

The main idea is simple: If we disregard the signs of the terms, the $\operatorname{Pfaffian} \operatorname{Pf}(G)$, by definition, encompasses the same terms as the generating function $M_{G}$ of matchings in $G$. 
Figure 9: Illustration for Corollary 2. Consider the ordered set of vertices

$$
V=\left\{a_{1}, a_{2}, a_{3} ; b_{1}, b_{2}, \ldots, b_{7}\right\} .
$$

The picture shows the matching

$$
\rho=\left\{\left\{a_{1}, b_{5}\right\},\left\{a_{2}, b_{2}\right\},\left\{a_{3}, b_{7}\right\},\left\{b_{1}, b_{4}\right\},\left\{b_{3}, b_{6}\right\}\right\}
$$

in $S_{A, B}$, where $A=\left\{a_{1}, a_{2}, a_{3}\right\}$ and $B=\left\{b_{1}, b_{2}, \ldots, b_{7}\right\}$.

Let $Y=\left\{b_{2}, b_{5}, b_{7}\right\}$ and observe that $\rho$ may be viewed as superposition of the red matching $\mu=\left\{\left\{a_{1}, b_{5}\right\},\left\{a_{2}, b_{2}\right\},\left\{a_{3}, b_{7}\right\}\right\}$ in $K_{A, Y}$ and the blue matching $\nu=\left\{\left\{b_{1}, b_{4}\right\},\left\{b_{3}, b_{6}\right\}\right\}$ in $K_{B \backslash Y}$ (blue edges are drawn as dashed lines). All crossings in $\rho$ are indicated by circles; the crossings which are not present in $\mu$ or in $\nu$ are indicated by two concentric circles.

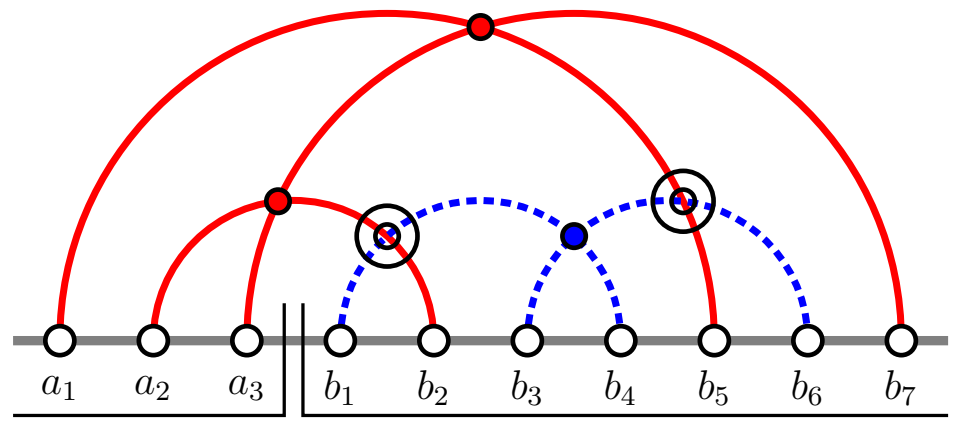

So if it is possible to modify the weight function $\omega$ by introducing signs such that for all matchings $\mu$ in $G$ the modified weight function $\omega^{\prime}$ is

$$
\omega^{\prime}(\mu)=\operatorname{sgn}(\mu) \omega(\mu),
$$

then the Pfaffian (for the modified weight-function $\omega^{\prime}$ ) would be equal to $M_{G}$ (for the original weight-function $\omega)$.

Such modification $\omega \rightarrow \omega^{\prime}$ could be described as follows. Let $G$ be some graph with weight function $\omega$ and assume some orientation $\xi$ on the pairs of vertices of $G$ :

$$
\xi: \mathrm{V}(G) \times \mathrm{V}(G) \rightarrow\{1,-1\} \text { such that } \xi(v, u)=-\xi(u, v)
$$

Consider the skew-symmetric square matrix $D(G, \xi)$ with row and column indices corresponding to the ordered set of vertices $\mathrm{V}(G)=\left\{v_{1}, \ldots, v_{n}\right\}$, and entries

$$
\begin{aligned}
& d_{i, i}=0, \\
& d_{i, j}=\xi\left(v_{i}, v_{j}\right) \times \sum_{\substack{e \in \mathrm{E}(G), e=\left\{v_{i}, v_{j}\right\}}} \omega(e) \text { for } i \neq j .
\end{aligned}
$$

Clearly, the weights $\omega^{\prime}(\mu)$ of the terms in the $\operatorname{Pfaffian} \operatorname{Pf}(D(G, \xi))$ differ from the weights $\omega(\mu)$ of the terms in the $\operatorname{Pfaffian} \operatorname{Pf}(G)$ (i.e., for $G$ without orientation) only by a sign 
which depends on the orientation $\xi$. So if we find an orientation $\xi$ of $G$ under which all the terms in the Pfaffian $\operatorname{Pf}(D(G, \xi))$ have the same sign $(-1)^{m}$, i.e., for all matchings $\mu$ of $G$ we have

$$
\operatorname{sgn}(\mu) \omega^{\prime}(\mu)=(-1)^{m} \omega(\mu),
$$

then the generating function $M_{G}$ is equal to $(-1)^{m} \operatorname{Pf}(D(G, \xi))$.

All terms in the Pfaffian $\operatorname{Pf}(D(G, \xi))$ have the same sign if and only if all the terms in the squared Pfaffian $\operatorname{Pf}(D(G, \xi))^{2}$ have the positive sign, which by Cayley's theorem (stated previously as Theorem 1$)$ is equivalent to all the terms in the $\operatorname{determinant} \operatorname{det}(D(G, \xi))$ being positive. According to Step 1 of the proof of Cayley's theorem, the non-vanishing terms in this determinant correspond to permutations $\pi$ with cycle decompositions where every cycle has even length. Since an even-length cycle contributes the factor $(-1)$ to the sign of $\pi$, i.e.,

$$
\operatorname{sgn}(\pi)=(-1)^{\text {number of even-length cycles in } \pi},
$$

the overall sign of the term in the determinant certainly will be positive if the weight of each even-length cycle contributes an offsetting factor $(-1)$, i.e., if every even-length cycle in $\pi$ contains an odd number of elements $d_{i, \pi(i)}$ with negative sign. Note that this condition is always fulfilled for cycles of length 2: Exactly one of the elements in $\left(d_{i, j}, d_{j, i}\right)$ has the negative sign.

These considerations can be restated in terms of the graph $G$.

Definition 2. Let $G$ be some graph with weight function $\omega$ and orientation $\xi$. The superposition of two arbitrary matchings of $G$ yields a covering of the bicoloured graph $B=G_{\mathbf{r} \mid \mathbf{b}}, \mathbf{r}=\mathbf{b}=\emptyset$ (i.e., there are no coloured vertices), with even-length cycles. (Recall that a superposition of matchings in $G$ corresponds to a term in the squared Pfaffian $\operatorname{Pf}(D(G, \xi))^{2}$, and the corresponding covering with even-length cycles corresponds to a term in the determinant $\operatorname{det}(D(G, \xi))$; see the proof of Cayley's theorem.)

$A$ cycle in $B$ of length $>2$, which arises from the superposition of two matchings of $G$, corresponds to a "normal" even-length cycle $C$ in $G$ : We call such cycle $C$ a superposition cycle.

An oriented edge $e=(v, w)$ in $G$ is called $\mathrm{co}^{-}$oriented with $\xi$, if $\xi(v, w)=1$; otherwise, e is called contra-oriented. The orientation $\xi$ is called admissible if every superposition cycle $C$ contains an odd number of co-oriented edges (and an odd number of contra-oriented edges, since $C$ has even length) with respect to some arbitrary but fixed orientation of $C$.

These considerations can be summarized as follows [8, Theorem [1] on page 92]:

Theorem 2 (Kasteleyn). Let $G$ be a graph with weight function $\omega$. If $G$ has an admissible orientation $\xi$, then the total weight of all matchings of $G$ equals the Pfaffian of $D(G, \xi)$ up to sign:

$$
M_{G}= \pm \operatorname{Pf}(D(G, \xi))
$$


Figure 10: Decomposition of a graph in 2-connected blocks, bridges and isolated vertices. The cut-vertices are drawn as black circles. The graph shown here is planar, each of its three 2-connected blocks consists of a single cycle. The clockwise orientation of these cycles (in the given embedding) is indicated by grey arrows.

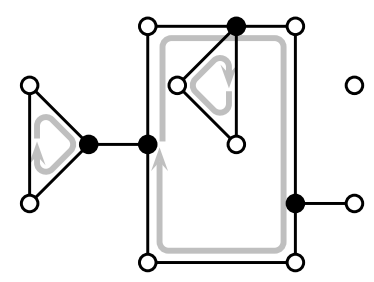

\subsection{Admissible orientations for planar graphs}

While the existence of an admissible orientation is not guaranteed in general, for a planar graphs $G$ such orientation can be constructed [8].

For this construction, we need some facts from graph theory. Let $G$ be a graph. If there are two different vertices $p \neq q \in \mathrm{V}(G)$ belonging to the same connected component $G^{\star}$ of $G$, such that there is no cycle in $G^{\star}$ that contains both vertices $p$ and $q$ (i.e., $G^{\star}$ is not 2-connected), then by Menger's Theorem (see, e.g., [4, Theorem 3.3.1]) there exists a vertex $v$ in $G^{\star}$ such that $\left[G^{\star}-\{v\}\right]$ is disconnected: Such vertex $v$ is called an articulation vertex or cutvertex. The whole graph $G$ is subdivided by its cutvertices, in the following sense: Each cutvertex connects two or more blocks, i.e., maximal connected subgraphs that do not contain a cutvertex. Such blocks are

- either maximal 2-connected subgraphs $H$ of $G$ (i.e., for every pair of different vertices $p, q \in \mathrm{V}(H)$ there exists a cycle in $H$ that contains both $p$ and $q$ ),

- or single edges (called bridges),

- or isolated vertices.

See Figure 10 for an illustration.

Since we deal with cycles here, we are mainly interested in the the 2-connected blocks which are not isolated vertices. (Clearly, a graph with an isolated vertex has no matching.)

In the following, assume that $G$ is planar and consider an arbitrary but fixed embedding of $G$ in the plane: So from now on, when we speak of $G$ we always mean " $G$ in its fixed planar embedding".

For every 2-connected block $H$ of $G$, consider the embedding "inherited" from $G$. Note that the boundary of a face of a 2-connected planar graph always is a cycle. 
Figure 11: Contour cycles are not the boundary of faces, but the boundary of the closure of faces. The pictures show three copies of graph $G$ (in its fixed embedding): Thick grey lines indicate the boundary of face $F_{3}$ in the left picture, the contour cycle corresponding to face $F_{3}$ in the middle picture, and the cycle encircling faces $F_{1}, F_{2}$ and $F_{3}$ in the right picture.
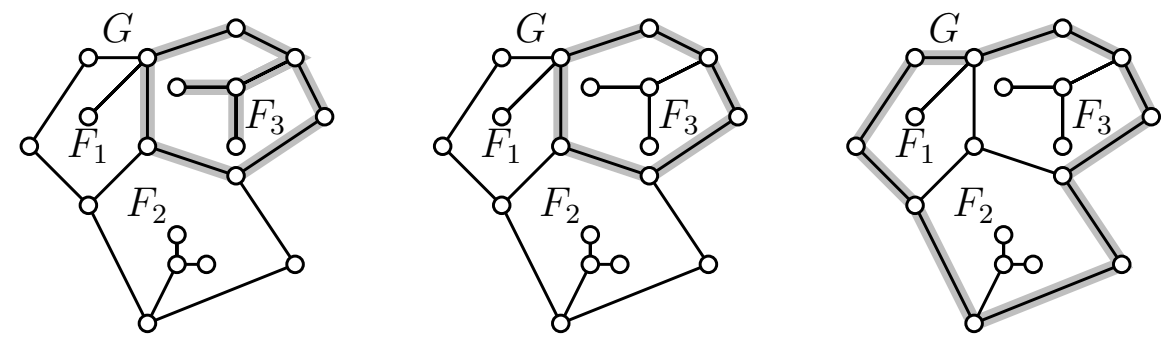

Let $F$ be some bounded face of $G$ (we shall never consider the unbounded face of the graphs here). The closure of $F$ corresponds to a face $F_{H}$ in a 2 -connected block $H$ of $G$ (looking at $H$ alone, forgetting the rest of $G$ ). The boundary of $F_{H}$ appears as a cycle in $G$ : Such cycle is called a contour cycle in $G$ (in general, this is not the boundary of $F$ in $G$, see Figure 11). The vertices of $G$ lying in the interior of some cycle $C$ of $G$ (i.e., lying in the bounded region confined by $C$ in the fixed embedding) are called the interior vertices of $C$. For the number of interior vertices of $C$ we introduce the notation $|C|^{\circ}$.

In the plane, consider the clockwise orientation: This determines a unique orientation for every cycle of $G$ (by choosing a "center" in the bounded region confined by $C$ in the fixed embedding of $G$ and traversing the edges of $C$ in the clockwise orientation around this center, see Figure 10). For an arbitrary orientation $\xi$ of the edges of $G$, we denote by $|C|^{\circlearrowright}$ the number of $c o$-oriented edges of $C$ (with respect to the clockwise orientation and $\xi$ ). We call $\xi$ a balanced orientation, if for every contour cycle $C$ of $G$ there holds

$$
|C|^{\circlearrowright}+|C|^{\circ} \equiv 1 \quad(\bmod 2)
$$

Then we have $[8$, Lemma $[2 a]$ on page 93$]$ :

Lemma 1. For each finite planar graph $G$ there is a balanced orientation $\xi$.

Proof. For bridges (edges not belonging to a 2 -connected block) in $G$, we may choose an arbitrary orientation.

For the remaining edges, we may construct the orientation by considering independently the 2-connected blocks $H$ of $G$, one after another.

So let $H$ be a 2 -connected block with the embedding inherited from $G$. Look at $H$ alone, i.e., forget the rest of $G$. The algorithm is as follows: 
Start by choosing an arbitrary contour cycle $C_{1}$ in $H$ and choose an orientation for its edges such that the number of co-oriented edges of $C_{1}$ and the number of interior vertices of $C_{1}$ (viewed as a cycle in $G$ ) are of opposite parity (clearly, this is possible). Note that in the inherited embedding of $H$, the union of $C_{1}$ and the face bounded by $C_{1}$ is a simply connected region in the plane (homeomorphic to the closed disk).

Now repeat the following step until all edges of $H$ are oriented: Assume that the edges belonging to contour cycles $C_{1}, C_{2}, \ldots, C_{n}$ have already been oriented by our algorithm and that the union of all these cycles and corresponding faces is a simply connected region in the plane. Choose a contour cycle $C_{n+1}$ that contains at least one edge which is not yet oriented, such that the union of the cycles $C_{1}, C_{2}, \ldots, C_{n+1}$ together with their corresponding faces is again a simply connected region in the plane. (A moment's thought shows that this is possible.) Clearly, for the edges of $C_{n+1}$ which are not yet oriented, we can choose an orientation such that the number of co-oriented edges of $C_{n+1}$ and the number of interior vertices of $C_{n+1}$ (viewed as a cycle in $G$ ) are of opposite parity.

It is clear that every cycle in the planar graph $G$ encircles one or more faces (see Figure 11). We use this simple fact to give the following generalization of Lemma 1 [8, Lemma [2b] on page 93]:

Proposition 2. Let $G$ be a finite planar graph with balanced orientation $\xi$. Then we have for every cycle $C$ of $G$ (not only contour cycles!)

$$
|C|^{\circlearrowright}+|C|^{\circ} \equiv 1 \quad(\bmod 2) \text {. }
$$

Proof. We prove this by induction on the number $n$ of faces encircled by the cycle $C$ : For $n=1$, the assertion is true by definition ( $\xi$ is balanced).

So assume the assertion to be true for all cycles encircling $n$ faces, and consider some cycle $C$ encircling $n+1$ faces. Select one of these faces $F_{i}$ (the interior of some contour cylce $C_{i}$ ) such that the union of all other faces $\bigcup_{j=1, j \neq i}^{n+1} F_{j}$ (together with their corresponding contour cycles $C_{j}$ ) is also a simply connected region: A moment's thought shows that this is always possible.

For the cycle $C^{\prime}$ encircling $\bigcup_{j=1, j \neq i}^{n+1} F_{j}$ and for the cycle $C_{i}$, the assertion is true by induction. By construction, the edges belonging to both $C^{\prime}$ and $C_{i}$ form a path of length $k>0$,

$$
\left(v_{0}, v_{1}, \ldots, v_{k}\right)
$$

and the new interior vertices of $C$ (which are not also interior vertices of $C_{i}$ or $C^{\prime}$ ) are precisely $v_{1}, \ldots v_{k-1}$. Now observe that for every edge $e$ of $p$ we have: If $e$ is co-oriented in $C_{i}$, then it is contra-oriented in $C^{\prime}$, and vice versa. Hence we have

$$
\begin{aligned}
& |C|^{\circlearrowright}=\left|C^{\prime}\right|^{\circlearrowright}+\left|C_{i}\right|^{\circlearrowright}-k, \\
& |C|^{\circ}=\left|C^{\prime}\right|^{\circ}+\left|C_{i}\right|^{\circ}+k-1 .
\end{aligned}
$$

This proves the assertion. 
Now we obtain immediately the following result [8, Theorem [2] on page 94]:

Theorem 3 (Kasteleyn). A balanced orientation for a finite planar graph $G$ is admissible. Therefore, for every finite planar graph $G$ there exists an admissible orientation.

Proof. Simply observe that for a superposition cycle $C$ of a planar graph the number of interior points is necessarily even. (Recall that different superposition cycles cannot have a vertex in common.) So the assertion follows from Proposition 2.

Observe that the "balancedness" (and hence the admissibility) of the orientation is "inherited" by certain induced subgraphs:

Corollary 3. Let $G$ be a finite planar graph with balanced orientation $\xi$. Let $C$ be some contour cycle of $G$, and let $S=\left\{v_{1}, \ldots, v_{2 k}\right\}$ be some set of $2 k$ vertices of $C$. Consider $G^{\prime}=[G-S]$ with the orientation $\xi$ inherited from $G$ : Then $\xi$ is balanced for $G^{\prime}$.

Proof. Simply note that the face encircled by $C$ in $G$ belongs to a bigger face $F$ of $G^{\prime}$, and the condition (16) holds true for all contour cycles corresponding to the other faces of $G^{\prime}$ (since they are also contour cycles in $G$, and nothing changed for them).

If $F$ is the unbounded face in $G^{\prime}$, then the condition (16) for $C$ in $G$ simply vanished in $G^{\prime}$.

Else the contour cycle of $F$ in $G^{\prime}$ is a cycle $C^{\prime}$ in $G$, for which $\left|C^{\prime}\right|^{\circlearrowright} \not \equiv\left|C^{\prime}\right|^{\circ}(\bmod 2)$ holds in $G$ and in $G^{\prime}$ by Proposition 2 (since the number of interior points of $C^{\prime}$ is decreased by $2 k$ in $G^{\prime}$.

So it seems that Proposition 1 gives an identity for special Pfaffians which correspond to planar graphs $G$ : Let $\xi$ be an admissible orientation for $G$, and assume the same (inherited) orientation for induced subgraphs of $G$, then (1) translates to

$$
\begin{aligned}
& \pm \operatorname{Pf}(D(G, \xi)) \cdot \operatorname{Pf}(D([G-\{a, b, c, d\}], \xi)) \\
& \pm \operatorname{Pf}(D([G-\{a, c\}], \xi)) \cdot \operatorname{Pf}(D([G-\{b, d\}], \xi))= \\
& \pm \operatorname{Pf}(D([G-\{a, b\}], \xi)) \cdot \operatorname{Pf}(D([G-\{c, d\}], \xi)) \\
& \quad \pm \operatorname{Pf}(D([G-\{a, d\}], \xi)) \cdot \operatorname{Pf}(D([G-\{b, c\}], \xi))
\end{aligned}
$$

for the "proper" choice of signs, by Kasteleyn's Theorem (stated here as Theorem 2).

But it turns out that the "proper" choice of signs is "always +" or "always -", and for constant sign + or,$-(17)$ is in fact an identity for Pfaffians in general, namely the special case [9, Equation (1.1)] of an identity [9, Equation (1.0)] due to Tanner [19]. This can be made precise as follows: 
Definition 3. Assume an identity for Pfaffians of the form

$$
\sum_{i=1}^{m} \operatorname{Pf}\left(G_{i}\right) \cdot \operatorname{Pf}\left(H_{i}\right)=\sum_{j=1}^{n} \operatorname{Pf}\left(G_{j}^{\prime}\right) \cdot \operatorname{Pf}\left(H_{j}^{\prime}\right),
$$

where all the graphs $G_{i}, H_{i}, G_{j}^{\prime}$ and $H_{j}^{\prime}$ involved are induced subgraphs of some "supergraph" G, with

$$
\mathrm{V}\left(G_{i}\right) \cup \mathrm{V}\left(H_{i}\right)=\mathrm{V}\left(G_{j}^{\prime}\right) \cup \mathrm{V}\left(H_{j}^{\prime}\right)=\mathrm{V}(G)
$$

for $i=1, \ldots, m$ and $j=1, \ldots, n$. If this identity comes, in fact, from a sign- and weight-preserving involution which maps

- the family of superpositions of matchings corresponding to the left-hand side

- to the family of superpositions of matchings corresponding to the right-hand side,

then we say that the identity is of the involution-type.

Remark 1. An identity of the involution-type could, of course, be written in the form

$$
\sum_{i=1}^{m} \operatorname{Pf}\left(G_{i}\right) \cdot \operatorname{Pf}\left(H_{i}\right)-\sum_{j=1}^{n} \operatorname{Pf}\left(G_{j}^{\prime}\right) \cdot \operatorname{Pf}\left(H_{j}^{\prime}\right)=0 .
$$

If we view the left-hand side as the generating function of signed weights of superpositions of matchings

$$
(-1)^{d} \cdot \operatorname{sgn}(\mu) \cdot \operatorname{sgn}(\nu) \cdot \omega(\mu) \cdot \omega(\nu),
$$

where

- $d=0$ for the objects corresponding to the unprimed pairs $\left(G_{i}, H_{i}\right)$

- and $d=1$ for the objects corresponding to the primed pairs $\left(G_{j}^{\prime}, H_{j}^{\prime}\right)$,

then the involution according to Definition 3 would appear as sign-reversing.

Lemma 2. If an identity for Pfaffians is of the involution-type, and if this identity can be specialized in a way such that

- the "supergraph" $G$ is planar with admissible orientation $\xi$,

- and the inherited orientation $\xi$ is also admissible for all the induced subgraphs $G_{i}$, $H_{i}(i=1, \ldots, m)$ and $G_{j}^{\prime}, H_{j}^{\prime}(j=1, \ldots, n)$ of $G$,

then this identity "translates" immediately to the corresponding identity for matchings, i.e., to

$$
\sum_{i=1}^{m} M_{G_{i}} \cdot M_{H_{i}}=\sum_{j=1}^{n} M_{G_{j}^{\prime}} \cdot M_{H_{j}^{\prime}}
$$


Proof. Construct the graph $J$ with vertex set

$$
\underbrace{\left\{\left(G_{1}, H_{1}\right),\left(G_{2}, H_{2}\right), \ldots,\left(G_{m}, H_{m}\right)\right\}}_{\text {unprimed vertices }} \dot{ن} \underbrace{\left\{\left(G_{1}^{\prime}, H_{1}^{\prime}\right),\left(G_{2}^{\prime}, H_{2}^{\prime}\right), \ldots,\left(G_{n}^{\prime}, H_{n}^{\prime}\right)\right\}}_{\text {primed vertices }} .
$$

Connect two vertices $\left(G_{i}, H_{i}\right)$ and $\left(G_{j}^{\prime}, H_{j}^{\prime}\right)$ of $J$ by an edge if and only if the involution maps some superposition of matchings from $\left(G_{i}, H_{i}\right)$ to some superposition of matchings from $\left(G_{j}^{\prime}, H_{j}^{\prime}\right)$. Clearly, $J$ is bipartite; the bipartition is given by the sets of primed and unprimed vertices.

Recall that all the superpositions of matchings in the identity which correspond to some fixed vertex of $J$ have the same sign, since the inherited orientations are admissible. Let $Z$ be an arbitrary connected component of $J$ : It is easy to see that all the terms in the identity corresponding to vertices of $Z$ have the same sign. So cancelling the sign, we see that the total weight (without sign!) of the unprimed vertices of $Z$ equals the total weight (without sign!) of the primed vertices of $Z$, i.e., $Z$ corresponds to an identity for matchings.

This consideration can be applied to all connected components of $J$, and the sum of the corresponding identities for matchings gives the desired translation of the Pfaffian identity of the involution-type.

In the following, we shall say that an identity for matchings of planar graphs follows from an identity for Pfaffians by the Kasteleyn-Percus method, if it can be obtained by the "translation" in the sense of Lemma 2.

\section{Overlapping Pfaffian identities}

We consider Pfaffian identities here which are all of the involution-type: This fact will be obvious immediately from the proofs we give, since they are all based on weight-preserving involutions.

The following theorem is due to H.W.L. Tanner [19] (see [9, Equation (1.0)].

Theorem 4. Let $\alpha=\left(\alpha_{1}, \ldots, \alpha_{n}\right)$ and $\beta=\left(\beta_{1}, \ldots, \beta_{m}\right)$ be subsets of the ordered set $\gamma=\left(\alpha_{1}, \ldots, \alpha_{n}, \beta_{1}, \ldots, \beta_{m}\right)$. Let $k, 1 \leqslant k \leqslant m$, be arbitrary but fixed. Then there holds

$$
\operatorname{Pf}(\alpha) \operatorname{Pf}(\alpha \cup \beta)=(-1)^{k} \cdot \sum_{\substack{j=1, j \neq k}}^{m}(-1)^{j-1} \operatorname{Pf}\left(\alpha \cup\left\{\beta_{k}, \beta_{j}\right\}\right) \operatorname{Pf}\left((\alpha \cup \beta) \backslash\left\{\beta_{k}, \beta_{j}\right\}\right) .
$$

(Recall that all subsets inherit the order of $\gamma$. )

This identity is of the involution-type. 
Observe that for the special case $\beta=(a, b, c, d)$, (18) reads

$$
\begin{aligned}
& \operatorname{Pf}(\alpha) \cdot \operatorname{Pf}(\alpha \cup(a, b, c, d))+\operatorname{Pf}(\alpha \cup(a, c)) \cdot \operatorname{Pf}(\alpha \cup(b, d))= \\
& \operatorname{Pf}(\alpha \cup(a, b)) \cdot \operatorname{Pf}(\alpha \cup(c, d))+\operatorname{Pf}(\alpha \cup(a, d)) \cdot \operatorname{Pf}(\alpha \cup(b, c)),
\end{aligned}
$$

which implies (17) by the Kasteleyn-Percus method (simply set $\alpha:=\mathrm{V}(G) \backslash \beta$ ).

Tanner's identity (and the fact that it is of the involution-type) is obtained immediately from the following generalization (and its bijective proof) which Hamel [6, Theorem 2.1] attributes to Ohta [14]; it was also found by Wenzel (see [21, Proposition 2.3] and [5, Theorem 1]).

For arbitrary sets $A$ and $B$, denote the symmetric difference of $A$ and $B$ by

$$
A \triangle B:=(A \cup B) \backslash(A \cap B) \text {. }
$$

Theorem 5 (Ohta). Assume that the ordered set $\gamma=\left(v_{1}, v_{2}, \ldots, v_{n}\right)$ appears as $\gamma=\alpha \cup \beta$ with $\alpha \Delta \beta=\left(v_{i_{1}}, \ldots, v_{i_{t}}\right)$. Then we have:

$$
\sum_{\tau=1}^{t}(-1)^{\tau} \cdot \operatorname{Pf}\left(\alpha \Delta\left\{v_{i_{\tau}}\right\}\right) \cdot \operatorname{Pf}\left(\beta \Delta\left\{v_{i_{\tau}}\right\}\right)=0 .
$$

(Recall again that all subsets inherit the order of $\gamma$. )

This identity is of the involution-type.

The following proof given by Krattenthaler [10] again uses superposition of matchings, together with proper accounting for the sign-changes associated to swapping colours in bicoloured paths. We state this sign-change as follows:

Lemma 3. Consider the complete graph $K_{V}$ on the ordered set of vertices $V$, given as disjoint union of red, blue and white vertices

$$
V=\mathbf{b} \cup \mathbf{r} \cup \mathbf{w}=\left(v_{1}, v_{2}, \ldots, v_{n}\right),
$$

where $|\mathbf{b}| \equiv|\mathbf{r}|(\bmod 2)$. Draw the edges of $K_{V}$ in the specific way described in Definition 1. Let $B$ be the corresponding bicoloured graph, let $S=\mu \cup \nu$ be a superposition of matchings in $B$ (recall Observation 1), and let $p$ be a bicoloured path in $S$ (recall Observation 2) with end vertices $v_{i}$ and $v_{j}$. Swapping colours in $p$ (recall Observation 3) gives a superposition of matchings $S=\mu^{\prime} \cup \nu^{\prime}$ in a bicoloured graph $B^{\prime}$, and we have

$$
\operatorname{sgn}(\mu) \cdot \operatorname{sgn}(\nu)=(-1)^{\#\left(\text { vertices of }(\mathbf{b} \cup \mathbf{r}) \text { between } v_{i} \text { and } v_{j}\right)} \operatorname{sgn}\left(\mu^{\prime}\right) \cdot \operatorname{sgn}\left(\nu^{\prime}\right) .
$$

If $v_{i}$ and $v_{j}$ appear in the ordered set of coloured vertices

$$
\mathbf{c}=\mathbf{b} \cup \mathbf{r}=\left(w_{1}, w_{2}, \ldots, w_{|\mathbf{c}|}\right)
$$

as $v_{i}=w_{x}$ and $v_{j}=w_{y}$, then this amounts to

$$
\operatorname{sgn}(\mu) \cdot \operatorname{sgn}(\nu)=(-1)^{y-x+1} \operatorname{sgn}\left(\mu^{\prime}\right) \cdot \operatorname{sgn}\left(\nu^{\prime}\right) .
$$


Proof. According to Observation 4, the change in sign corresponding to removing some single arc $\left\{v_{k}, v_{l}\right\}$ from $\mu$ and adding it to $\nu$ amounts to

$$
\begin{aligned}
& (-1)^{\#\left(\text { vertices of }(\mathbf{b} \cup \mathbf{w}) \text { between } v_{k} \text { and } v_{l}\right)-\#\left(\text { vertices of }(\mathbf{r} \cup \mathbf{w}) \text { between } v_{k} \text { and } v_{l}\right)}= \\
& (-1)^{\#\left(\text { vertices of }(\mathbf{b} \cup \mathbf{r}) \text { between } v_{k} \text { and } v_{l}\right)} .
\end{aligned}
$$

Recolouring all the arcs in path $p$ with end vertices $v_{i}$ and $v_{j}$ thus gives a change in sign equal to the product of all these single sign-changes, which clearly amounts to

$$
(-1)^{\#\left(\text { vertices of }(\mathbf{b} \cup r) \text { between } v_{i} \text { and } v_{j}\right)} \text {. }
$$

Proof of Theorem 5. For the combinatorial interpretation of the left-hand side of (19), simply combine Observation 1 (superposition of matchings) with the definition of Pfaffians as given in Definition 1: after expansion of the products of Pfaffians, the typical summand is of the form

$$
(-1)^{\tau} \cdot \operatorname{sgn}(\mu) \cdot \operatorname{sgn}(\nu) \cdot \omega(\mu) \cdot \omega(\nu),
$$

where $(\mu, \nu)$ can be interpreted as superposition of matchings in the bicoloured graph $G_{\mathbf{r} \mid \mathbf{b}}$ derived from the complete graph $G$ on vertices $\alpha \cup \beta$, with

$$
\begin{aligned}
& \text { - } \mathbf{b}=(\alpha \backslash \beta) \Delta\left\{v_{i_{\tau}}\right\}, \\
& \text { - } \mathrm{r}=(\beta \backslash \alpha) \Delta\left\{v_{i_{\tau}}\right\} .
\end{aligned}
$$

According to Observation 1, the operation $\chi_{v}$ of swapping colours in the unique path $p$ starting in vertex $v=v_{i_{\tau}}$ is an involution which preserves the weight $\omega(\mu) \cdot \omega(\nu)$.

So the proof is complete if we can show that $\chi_{v}$ is in fact sign-reversing in the following sense. Note that $\chi_{v}$ yields a superposition of matchings $\left(\mu^{\prime}, \nu^{\prime}\right)$ in the bicoloured graph $G_{\mathbf{r}^{\prime} \mid \mathbf{b}^{\prime}}$ with

- $\mathbf{b}^{\prime}=(\alpha \backslash \beta) \triangle\left\{v_{i_{\rho}}\right\}$,

$$
\text { - } \mathrm{r}^{\prime}=(\beta \backslash \alpha) \Delta\left\{v_{i_{\rho}}\right\} \text {, }
$$

where $v_{i_{\rho}}$ is the other endpoint of the unique path $p$. Thus we have to show

$$
(-1)^{\tau} \cdot \operatorname{sgn}(\mu) \cdot \operatorname{sgn}(\nu)=-(-1)^{\rho} \cdot \operatorname{sgn}\left(\mu^{\prime}\right) \cdot \operatorname{sgn}\left(\nu^{\prime}\right) \text {. }
$$

But this follows immediately from Lemma 3.

The same idea of proof applies to the following generalization, which to the best of my knowledge is due to Krattenthaler [10]: 
Theorem 6 (Krattenthaler). Assume that the ordered set $\gamma=\left(v_{1}, v_{2}, \ldots, v_{n}\right)$ appears as $\gamma=\alpha \cup \beta$. Let $M=\alpha \Delta \beta$ and $t=|M|$.

Then we have for all $s, 0 \leqslant s \leqslant\lfloor t / 2\rfloor$ :

$$
\sum_{\substack{Y \subseteq M \\|Y|=2 s+1}}(-1)^{\Sigma(Y \subseteq M)} \cdot \operatorname{Pf}(\alpha \Delta Y) \cdot \operatorname{Pf}(\beta \Delta Y)=0 .
$$

If $t>0$, then we have also

$$
\sum_{s=0}^{\lfloor t / 2\rfloor} \sum_{\substack{Y \subseteq M \\|Y|=2 s}}(-1)^{\Sigma(Y \subseteq M)} \cdot \operatorname{Pf}(\alpha \Delta Y) \cdot \operatorname{Pf}(\beta \Delta Y)=0
$$

Note that (21) is trivially true if $|\alpha|$ or $|\beta|$ is even; and that (22) is trivially true if $|\alpha|$ or $|\beta|$ is odd: The "interesting" instances of (21) and (21) appear for $|\alpha| \equiv|\beta| \equiv 1(\bmod 2)$ and $|\alpha| \equiv|\beta| \equiv 0(\bmod 2)$, respectively.

These identities are of the involution-type.

Proof. For every superposition of matchings $(\mu, \nu)$ involved in (21) (in the sense of the proof of Theorem 5) consider the subset $S \subseteq Y$ of vertices $v$ with the property that the other endpoint of the unique path starting in $v$ does not belong to $Y$. Note that $S$ is of odd cardinality, so in particular $S \neq \emptyset$.

By the same reasoning as in the proof of Theorem 5, simultaneously swapping colours in all paths starting in vertices from $S$ yields a sign-reversing and weight-preserving involution, which proves (21).

Now consider the family of superpositions of matchings corresponding to (22). Let $v$ be some arbitrary, but fixed element in $M$, and note that the operation $\chi_{v}$ of swapping colours in the unique path with starting point $v$ yields a weight-preserving involution, which is sign-reversing according to Lemma 3.

Since $\operatorname{Pf}(X) \equiv 0$ if the cardinality of $X$ is odd, we may restate (a weaker version of) the above theorem in a uniform way.

Corollary 4. Assume that the ordered set $\gamma=\left(v_{1}, v_{2}, \ldots, v_{n}\right)$ appears as $\gamma=\alpha \cup \beta$. Let $M=\alpha \Delta \beta \neq \emptyset$. Then we have:

$$
\sum_{Y \subseteq M}(-1)^{\Sigma(Y \subseteq M)} \cdot \operatorname{Pf}(\alpha \Delta Y) \cdot \operatorname{Pf}(\beta \Delta Y)=0 .
$$

This identity is of the involution-type. 


\subsection{Further applications}

For the rest of this paper, consider the complete graph $G=K_{V}$ with (ordered) vertex set $V=\left(v_{1}, v_{2}, \ldots, v_{n}\right)$, where $V$ appears as the disjoint union of coloured vertices and white vertices, $V=\mathbf{c} \cup \mathbf{U}$, and where the set of coloured vertices is partitioned into two sets of equal size $\mathbf{c}=\mathbf{R} \cup \mathbf{B},|\mathbf{R}|=|\mathbf{B}|$. For the following assertions, imagine that all vertices in $\mathbf{B}$ are "initially" blue and that all vertices in $\mathbf{R}$ are "initially" red, and bicoloured graphs are derived therefrom by changing this "initial" colouring.

\subsubsection{Srinivasan's result}

For the next Lemma, let $X$ be some fixed subset $X \subseteq \mathbf{B}$, and let $\mathbf{b}:=\mathbf{B} \backslash X$ and $\mathrm{r}:=\mathrm{R} \cup X$. Consider the set of all superpositions of matchings $\mu \cup \nu$ in $G_{\mathrm{r} \mid \mathbf{b}}$ with the additional property, that every bicoloured path in $\mu \cup \nu$ has at most one end vertex in B: denote this set by $\mathcal{F}(G \| X)$. To every object $\mu \cup \nu \in \mathcal{F}(G \| X)$ assign signed weight

$$
\omega(\mu \cup \nu):=(-1)^{\Sigma(B \backslash X \subseteq \mathbf{c})} \operatorname{sgn}(\mu) \cdot \operatorname{sgn}(\nu) \cdot \omega(\mu) \cdot \omega(\nu) .
$$

Now consider the generating function $F$ of $\bigcup_{X \subseteq B} \mathcal{F}(G \| X)$, i.e.,

$$
F:=\sum_{X \subseteq \mathbf{B}} \sum_{f \in \mathcal{F}(G \| X)} \omega(f) .
$$

Lemma 4. With the above definitions, we have for the generating function $F$ :

$$
F=(-1)^{\boldsymbol{\Sigma}(B \subseteq \mathbf{c})} \cdot \sum_{X \subseteq \mathbf{B}}(-1)^{\boldsymbol{\Sigma}(X \subseteq \mathbf{c})} \cdot \operatorname{Pf}(\mathbf{R} \cup \mathbf{w} \cup X) \cdot \operatorname{Pf}((\mathbf{B} \cup \mathbf{w}) \backslash X) .
$$

Proof. Clearly, all the signed weights of objects from $\bigcup_{X \subseteq \mathbf{B}} \mathcal{F}(G \| X)$ do appear in the sum on the right-hand side of (24). So we have to show that all the "superfluous" terms in (24) cancel.

These "superfluous" objects are precisely superpositions of matchings, where there exists a bicoloured path connecting two vertices of $\mathbf{B}$ : of all such paths choose the one, $p$, with the smallest end vertex, and swap colours in $p$. This gives a weight-preserving involution, which is sign-reversing according to Lemma 3.

From this, we easily deduce (a slight generalization of) a result of Srinivasan ([17, Corollary $3.2]$, see also [6, Theorem 3.3]):

Corollary 5 (Srinivasan). Let $m+n$ be an even integer and consider the ordered set $V=\left(v_{1}, \ldots, v_{m+n}\right)$, partitioned into the disjoint subsets $A=\left(v_{i_{1}}, \ldots, v_{i_{m}}\right)$ and $B=$ $\left(v_{j_{1}}, \ldots, v_{j_{n}}\right)$. Then we have the following expansions: 
If $m<n$, then

$$
\operatorname{Pf}(V)=-\sum_{\substack{X \subset B, X \neq B}}(-1)^{\Sigma(B \backslash X \subseteq V)} \cdot \operatorname{Pf}(A \cup X) \cdot \operatorname{Pf}(B \backslash X) .
$$

If $m=n$, then

$$
\operatorname{Pf}(V)=\operatorname{Pf}(A, B)-\sum_{\substack{X \subset B, X \neq B}}(-1)^{\Sigma(B \backslash X \subseteq V)} \cdot \operatorname{Pf}(A \cup X) \cdot \operatorname{Pf}(B \backslash X) .
$$

If $m>n$, then

$$
\begin{aligned}
& \operatorname{Pf}(V)=(-1)^{\left(\begin{array}{c}
n+1 \\
2
\end{array}\right)} \sum_{\substack{Y \subset A,|Y|=n}}(-1)^{\Sigma(Y \subseteq A)} \operatorname{Pf}(A \backslash Y) \cdot \operatorname{Pf}(B, Y) \\
&-\sum_{\substack{X \subset B, X \neq B}}(-1)^{\Sigma(B \backslash X \subseteq V)} \cdot \operatorname{Pf}(A \cup X) \cdot \operatorname{Pf}(B \backslash X) .
\end{aligned}
$$

Proof. We apply Lemma 4 with $\mathbf{R}=A, \mathbf{B}=B$ and $\mathbf{w}=\emptyset$ : since there are no white vertices, bicoloured paths in any superposition of matchings $(\mu \cup \nu) \in \bigcup_{X \subseteq B} \mathcal{F}(G \| X)$ are simply edges, whose end vertices are of the same colour: hence we must have $X=B$ (because there is no matching on $B \backslash X \neq \emptyset$ where every edge has only one end vertex in $B$ ). So $\mu \cup \nu$ corresponds to a unique matching in the semi-bipartite graph $S_{B, A}$, and vice versa, whence we have

$$
F=\operatorname{Pf}\left(S_{B, A}\right)
$$

Expanding the trivial identity

$$
\operatorname{Pf}(V)=\operatorname{Pf}\left(S_{B, A}\right)-(F-\operatorname{Pf}(A \cup B) \cdot \operatorname{Pf}(\emptyset))
$$

according to (13) and (24), respectively, proves all three assertions: note that $\operatorname{Pf}\left(S_{B, A}\right)=0$ if $m<n$, and $\operatorname{Pf}\left(S_{B, A}\right)=\operatorname{Pf}(A, B)$ if $m=n$.

\subsubsection{Graphical condensation}

For the rest of this paper, assume that $\mathbf{B}=\left(b_{1}, b_{2} \ldots, b_{k}\right)$ and $\mathbf{R}=\left(r_{1}, r_{2}, \ldots, r_{k}\right)$ and that the ordered set of coloured vertices appears as $\mathbf{c}=\left(r_{1}, b_{1}, r_{2}, b_{2} \ldots, r_{k}, b_{k}\right)$.

Definition 4 (Planar weight function). We call a weight function $\omega$ on $\mathrm{E}(G)$ a planar weight function if it assigns weight zero to every superposition of matchings $\mu \cup \nu$ in every $G_{\mathbf{r} \mid \mathbf{b}}$ (where $\mathbf{r} \dot{\cup} \mathbf{b}=\mathbf{R} \cup \dot{\mathbf{B}}$ is an arbitrary partition of the coloured vertices in two subsets of blue and red vertices) that contains a bicoloured path $p$ connecting two vertices from $\mathbf{B}$ or two vertices from $\mathbf{R}$. 
Lemma 5. In the setting described above, assume that the weight function is planar.

Then we have for all (fixed) subsets $X \subseteq \mathbf{R}$ :

$$
\begin{aligned}
\sum_{W \subseteq \mathbf{B}} \operatorname{Pf}((\mathbf{R} \cup \mathbf{w}) \cup W) \cdot \operatorname{Pf}((\mathbf{B} \cup \mathbf{w}) \backslash W) \\
\quad=\sum_{V \subseteq \mathbf{B}} \operatorname{Pf}(((\mathbf{R} \cup \mathbf{w}) \cup V) \backslash X) \cdot \operatorname{Pf}(((\mathbf{B} \cup \mathbf{w}) \backslash V) \cup X) .
\end{aligned}
$$

This identity is of the involution-type.

Proof. The left-hand side of (28) corresponds to superpositions of matchings $\mu \cup \nu$ of non-vanishing weight with red vertices $\mathbf{R} \cup W$ and blue vertices $\mathbf{B} \backslash W$, for some subset $W \subseteq \mathbf{B}$. The assumption implies that every bicoloured path $p$, which starts in some vertex $v \in \mathbf{R}$, must have its other end in B. So according to (20) (and the specific "alternating ordering" of $\mathbf{c}=\mathbf{B} \cup \mathbf{R}$ ), the operation $\chi_{v}$ of swapping colours in $p$ gives a weight- and sign-preserving bijection. Thus, swapping colours in all bicoloured paths with end vertex in the fixed subset $X=\left\{v_{1}, \ldots, v_{m}\right\} \subseteq \mathbf{R}$ gives a weight- and sign-preserving bijection $\chi:=\chi_{v_{1}} \circ \ldots \circ \chi_{v_{m}}$.

Denote by $Y$ the set of the other end vertices of these paths. Note that $Y \subseteq$ B by assumption and consider the set $V:=W \triangle Y$ : it is obvious that the image of $\chi$ is precisely the set of superpositions of matchings corresponding to the right hand side of (28).

This yields immediately the following generalization of a result by Yan, Yeh and Zhang [23, Theorem 2.2] (also given in Kuo [11, Theorem 2.1 and Theorem 2.3]):

Corollary 6. Let $G$ be a planar graph with vertices $r_{1}, b_{1}, \ldots, r_{k}, b_{k}$ appearing in that cyclic order on the boundary of a face of $G$. Let $\mathbf{R}=\left\{r_{1}, \ldots, r_{k}\right\}$ and $\mathbf{B}=\left\{b_{1}, \ldots, b_{k}\right\}$. Then we have for every fixed subset $X \subseteq \mathbf{R}$

$$
\sum_{W \subseteq \mathbf{B}} M_{[G-(\mathbf{B} \backslash W)]} M_{[G-(\mathbf{R} \cup W)]}=\sum_{V \subseteq \mathbf{B}} M_{[G-((\mathbf{B} \backslash V) \cup X)]} M_{[G-((\mathbf{R} \cup V) \backslash X)]} .
$$

Proof. Recall that no two different paths arising from some superposition of matchings can have a vertex in common. So the ordering of $\mathbf{R} \cup \mathbf{B}$ along the boundary of a face of the planar graph $G$ implies that no bicoloured path can have both end vertices in $\mathbf{R}$ or in $\mathbf{B}$, hence the weight function is planar and we may apply Lemma 5 . By the Kasteleyn-Percus method, the assertion follows from (28).

\subsubsection{Ciucu's matching factorization theorem}

In the same manner, we may prove Ciucu's matching factorization Theorem [3, Theorem 1.2], in the (equivalent) formulation given in [22, Theorem 2.2]. 
Lemma 6. In the setting described above, assume that the weight function is planar. Moreover, assume that the set of coloured vertices is partitioned into two subsets of equal size, $\mathbf{c}=U \dot{\cup} V,|U|=|V|$, such

- that there is no even-length path of non-vanishing weight connecting any two vertices $(x, y)$ with $x \in U$ and $y \in V$,

- and that there is no odd-length path of non-vanishing weight connecting any two vertices $(x, y)$ with $x, y \in U$ or $x, y \in V$.

Let $\mathbf{B}_{U}:=\mathbf{B} \cap U, \mathbf{B}_{V}:=\mathbf{B} \cap V, \mathbf{R}_{U}:=\mathbf{R} \cap U$ and $\mathbf{R}_{V}:=\mathbf{R} \cap V$. (Note that $\left|\mathbf{B}_{U}\right|=\left|\mathbf{R}_{V}\right|$ and $\left|\mathbf{B}_{V}\right|=\left|\mathbf{R}_{U}\right|$.) Then we have

$$
2^{k} \cdot \operatorname{Pf}\left(\mathbf{w} \cup \mathbf{B}_{U} \cup \mathbf{R}_{V}\right) \cdot \operatorname{Pf}\left(\mathbf{w} \cup \mathbf{B}_{V} \cup \mathbf{R}_{U}\right)=
$$

(In the summands of the right-hand side, $\bar{X}=V \backslash X$ and $\bar{Y}=U \backslash Y$.) This identity is of the involution-type.

Proof. First, consider an arbitrary superposition of matchings in the bicoloured graph $G_{\mathbf{b} \mid \mathbf{r}}$, where $\mathbf{b} \cup \dot{\mathrm{r}}$ is an arbitrary partition of the set $\mathbf{R} \cup \mathbf{B}$. Since

- bicoloured paths of even length have end vertices of different colour,

- and bicoloured paths of odd length have end vertices of the same colour,

the assumption implies that if $\omega(\mu \cup \nu) \neq 0$, then every bicoloured path in $\mu \cup \nu$ must connect (vertices from) $\mathbf{r} \cap U$ with $\mathbf{b} \cap U$, $\mathbf{b} \cap U$ with $\mathbf{b} \cap V$, $\mathbf{b} \cap V$ with $\mathbf{r} \cap V$, or $\mathbf{r} \cap V$ with $\mathrm{r} \cap U$. See the left picture in Figure 12, where the possible connections by bicoloured paths are indicated by arrows.

Now consider the product of Pfaffians in the left hand side of (29), i.e., choose $\mathbf{b}=\mathbf{B}_{U} \cup \mathbf{R}_{V}$ and $\mathbf{r}=\mathbf{B}_{V} \cup \mathbf{R}_{U}$. Note that in this case we have $\mathbf{r} \cap U=\mathbf{R}_{U}, \mathbf{r} \cap V=\mathbf{B}_{V}, \mathbf{b} \cap U=\mathbf{B}_{U}$ and $\mathbf{b} \cap V=\mathbf{R}_{V}$, and the assertion about the possible connections with bicoloured paths (as depicted in the left picture of Figure 12) would hold also without the additional assumption, since the weight function $\omega$ is planar.

Consider some arbitrary superposition of matchings $\mu \cup \nu$ in $G_{\mathbf{b} \mid \mathbf{r}}$. Swapping colours in an arbitrary subset of bicoloured paths in $\mu \cup \nu$ gives a unique superposition of matchings $\mu^{\prime} \cup \nu^{\prime}$ of the same weight and sign, which appears in the right hand side of (29). So let the bicoloured paths $\left(p_{1}, p_{2}, \ldots, p_{k}\right)$ of $\mu \cup \nu$ appear in the order implied by their smaller end vertex (in the order of the set of coloured vertices c) and consider the set 
Figure 12: Illustration to the proof of Lemma 6: The left picture shows the partition of the set of coloured vertices in four subsets, induced by the bipartitions $\mathbf{r} \dot{\cup} \mathbf{b}=U \dot{U} V$. The arrows indicate the only possible connections by bicoloured paths: for instance, no bicoloured path can connect a vertex from $\mathbf{r} \cap U$ with $\mathbf{b} \cap V$, since such paths must have even length, which is ruled out by the additional assumption in Lemma 6 . The right picture shows the partition of the set of coloured vertices in eight subsets, induced by the bipartitions $\mathbf{r}^{\prime} \cup \dot{\mathbf{b}^{\prime}}=U \dot{\cup} V=\mathbf{R} \dot{\cup} \mathbf{B}$, where $\mathbf{r}^{\prime}=\bar{X} \cup \bar{Y}$ and $\mathbf{b}^{\prime}=X \cup Y$. The arrows indicate the only possible connections between "wrongly coloured" vertices (these are the ones indicated by the inscribed square with the dashed boundary) by bicoloured paths: in particular, every bicoloured path must have either no "wrongly coloured" end vertex or two "wrongly coloured" end vertices.
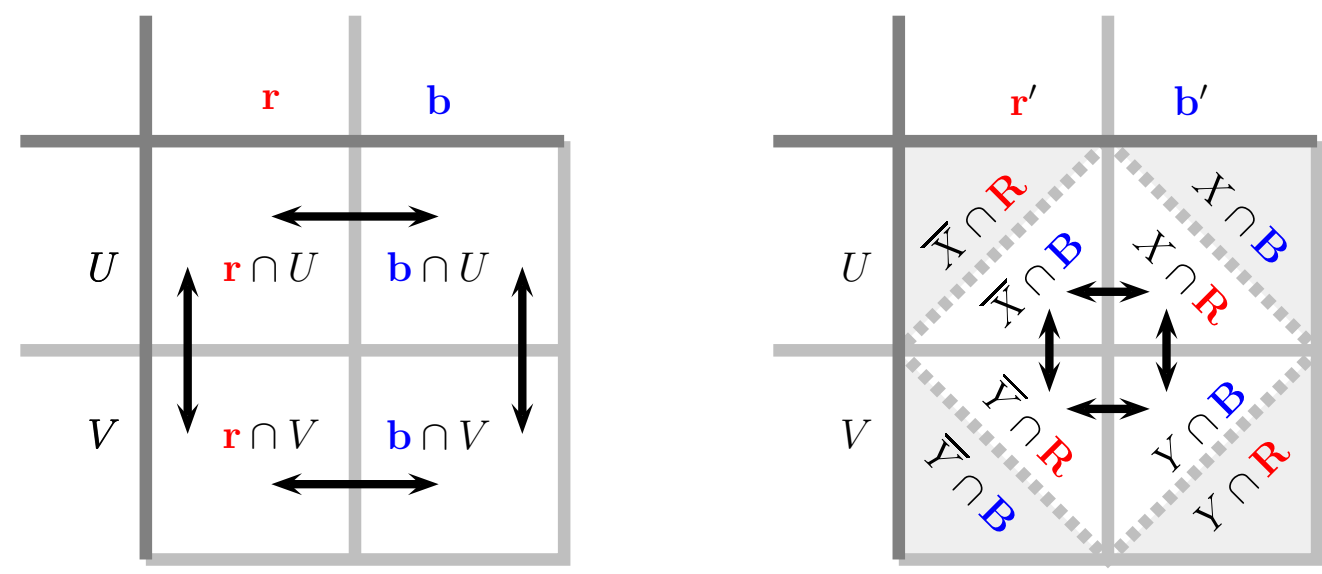
of pairs $(S, \mu \cup \nu)$, where $S=\left\{i_{1}, \ldots, i_{m}\right\}$ is a subset of $\{1, \ldots, k\}$. Clearly, this set of pairs corresponds to the left hand side of (29), and swapping colours in the bicoloured paths $p_{i_{1}}, \ldots, p_{i_{m}}$ defines a weight- and sign-preserving injective mapping $\chi$ into the set of superpositions of matchings corresponding to the right hand side of (29).

So it remains to show that this mapping is in fact surjective. To construct the preimage $\chi^{-1}\left(\mu^{\prime} \cup \nu^{\prime}\right)$ of some arbitrary superposition of matchings corresponding to the right hand side of (29), we would like to reverse the operation $\chi$, i.e.,

- note down the subset $S$ of paths (in the order described above) with "wrongly coloured" end vertices (these are vertices in $(\mathbf{B} \cap U) \cup(\mathbf{R} \cap V)$ which are red and vertices in $(\mathbf{B} \cap V) \cup(\mathbf{R} \cap U)$ which are blue $)$,

- and swap colours in all paths from $S$, hoping that this operation gives some superposition of matchings $\mu \cup \nu$ corresponding to the left hand side of (29).

This simple idea clearly will work if there is no path with only one "wrongly coloured" end vertex. To see that this is indeed the case, just have a look at the right picture in Figure 12: Since the weight function is planar, it is not possible for a superposition of matchings (of non-vanishing weight) to have only one "wrongly coloured" end vertex (for instance, there is no bicoloured connection from the "wrongly coloured" set $\bar{X} \cap \mathbf{B}$ to $\bar{Y} \cap \mathbf{B}$ or to $X \cap \mathbf{B}$ ). Thus $\chi$ is in fact a weight-preserving and sign-preserving bijection.

Corollary 7. Let $G$ be a planar bipartite graph, where the bipartition of the vertex set of $G$ is given as $\mathrm{V}(G)=A \cup \dot{\cup} B$. Assume that vertices $r_{1}, b_{1}, \ldots, r_{k}, b_{k}$ appear in that cyclic order on the boundary of a face of $G$, and let $\mathbf{R}=\left\{r_{1}, \ldots, r_{k}\right\}$ and $\mathbf{B}=\left\{b_{1}, \ldots, b_{k}\right\}$. Let $U:=A \cap(\mathbf{R} \cup \mathbf{B})$ and $V:=B \cap(\mathbf{R} \cup \mathbf{B})$, and assume that $|U|=|V|$.

Let $\mathbf{B}_{U}:=\mathbf{B} \cap U, \mathbf{B}_{V}:=\mathbf{B} \cap V, \mathbf{R}_{U}:=\mathbf{R} \cap U$ and $\mathbf{R}_{V}:=\mathbf{R} \cap U$. Then we have

$$
2^{k} \cdot M_{\left[G-\left(\mathbf{B}_{U} \cup \mathbf{R}_{V}\right)\right]} \cdot M_{\left[G-\left(\mathbf{B}_{V} \cup \mathbf{R}_{U}\right)\right]}=\sum_{\substack{X \subseteq V, Y \subseteq U \\|X|=|Y|}} M_{[G-(X \cup Y)]} \cdot M_{[G-(\bar{X} \cup \bar{Y})]} \cdot
$$

(In the summands of the right-hand side, $\bar{X}=V \backslash X$ and $\bar{Y}=U \backslash Y$.)

Proof. Note that the assumption that $G$ is bipartite implies the additional assumption of Lemma 6 . The assertion now follows from (29) by the Kasteleyn-Percus method.

\subsubsection{Graphical edge--condensation}

Consider again the situation of Lemma 5. Denote the edge connecting vertex $r_{i}$ with vertex $b_{i}$ by $e_{i}:=\left\{r_{i}, b_{i}\right\}$. From the given complete graph $G=K_{V}$ construct a new graph $G^{\prime}$ by replacing every edge $e_{i}$ by a path $\left(r_{i}, r_{i}^{\prime}, b_{i}^{\prime}, b_{i}\right)$ of length three, thus inserting 
Figure 13: Illustration: $G^{\prime}$ is obtained from $G$ by "subdividing" edge $e_{i}$, i.e., by replacing edge $e_{i}$ by the path $\left(r_{i}, r_{i}^{\prime}, b_{i}^{\prime}, b_{i}\right)$, thus introducing new vertices $r_{i}^{\prime}$ and $b_{i}^{\prime}$, and new edges $e_{i}^{r}:=\left\{r_{i}, r_{i}^{\prime}\right\}, e_{i}^{\prime}:=\left\{r_{i}^{\prime}, b_{i}^{\prime}\right\}$ and $e_{i}^{b}:=\left\{b_{i}^{\prime}, b_{i}\right\}$.

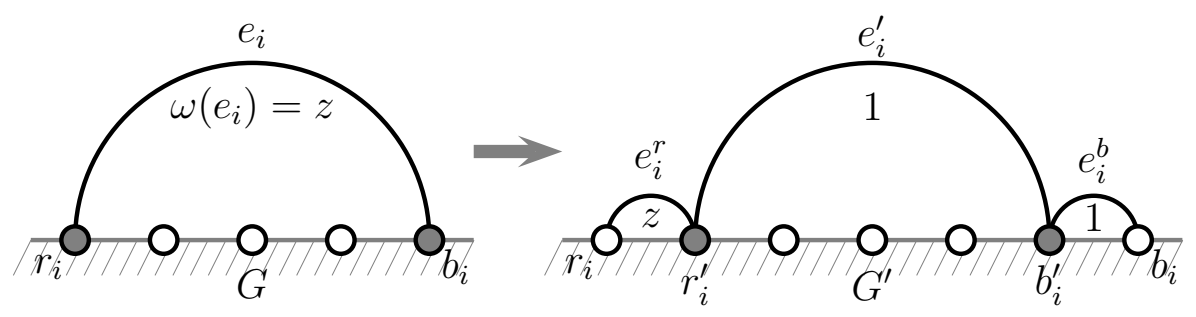

- new vertices $r_{i}^{\prime}$ and $b_{i}^{\prime}$, where $r_{i}^{\prime}$ is the immediate successor of $r_{i}$ and $b_{i}^{\prime}$ is the immediate predecessor of $b_{i}$, in the set of ordered vertices of $G^{\prime}$,

- and new edges $e_{i}^{r}:=\left\{r_{i}, r_{i}^{\prime}\right\}, e_{i}^{\prime}:=\left\{r_{i}^{\prime}, b_{i}^{\prime}\right\}$ and $e_{i}^{b}:=\left\{b_{i}^{\prime}, b_{i}\right\}$, where the weights of the new edges are given as $\omega\left(e_{i}^{r}\right)=\omega\left(e_{i}\right)$ and $\omega\left(e_{i}^{\prime}\right)=\omega\left(e_{i}^{b}\right)=1$.

Note that $G^{\prime}$ is not a complete graph $\left(r_{i}^{\prime}\right.$ and $b_{i}^{\prime}$ are vertices of degree 2 in $\left.G\right)$, but we may view it as a complete graph by introducing edges of weight zero. (Figure 13 illustrates this construction.)

Observe that there is an obvious weight-preserving bijection between matchings of $G$ and matchings of $G^{\prime}$ : For arbitrary $\mu \in \mathcal{M}_{G}$ and all $i=1, \ldots, k$,

- replace $e_{i}$ by edges $e_{i}^{r}$ and $e_{i}^{b}$ if $e_{i} \in \mu$,

- add $e_{i}^{\prime}$ to $\mu$ if $e_{i} \notin \mu$,

to obtain a matching $\mu^{\prime} \in \mathcal{M}_{G^{\prime}}$. Note that this bijection yields a change in sign equal to

$$
\prod_{i=1}^{k}(-1)^{\#\left(\text { vertices between } r_{i} \text { and } b_{i}\right)}=(-1)^{k+\Sigma(\mathbf{c} \subseteq V)},
$$

according to Observation 4 (since the edges $e_{i}^{r}$ and $e_{i}^{b}$ in $G^{\prime}$ can never be involved in any crossing). Thus we obtain immediately

$$
(-1)^{k+\Sigma(\mathrm{c} \subseteq V)} \operatorname{Pf}(G)=\operatorname{Pf}\left(G^{\prime}\right) .
$$

Now consider the bicoloured graph $B^{\prime}=G_{\mathbf{b}^{\prime} \mid \mathbf{r}^{\prime}}^{\prime}$, where $\mathbf{b}^{\prime} \dot{\cup} \mathrm{r}^{\prime}$ is some partition of the set of coloured vertices $\mathbf{c}^{\prime}:=\left\{r_{1}^{\prime}, b_{1}^{\prime}, \ldots, r_{k}^{\prime}, b_{k}^{\prime}\right\}$ in $G^{\prime}$.

If $r_{i}^{\prime}$ and $b_{i}^{\prime}$ are of different colours, then edges $e_{i}^{r}$ and $e_{i}^{b}$ must both belong to every superposition of matchings $\mu^{\prime} \cup \nu^{\prime}$ in the bicoloured graph $B^{\prime}=G_{\mathbf{b}^{\prime} \mid \mathbf{r}^{\prime}}^{\prime}$. So we may 
Figure 14: Illustration of the construction preceding Lemma 7: If $r_{i}^{\prime}$ and $b_{i}^{\prime}$ are of different colour, then $e_{i}^{r}$ and $e_{i}^{b}$ must necessarily both belong to every superposition of matchings $\mu^{\prime} \cup \nu^{\prime}$ and thus can be removed (after accounting for their weights, of course) together with the vertices $r_{i}^{\prime}$ and $b_{i}^{\prime}$. The resulting situation "locally" looks like the original graph $G$, with coloured vertices $r_{i}$ (of the same colour as $b_{i}^{\prime}$ ) and $b_{i}$ (of the same colour as $r_{i}^{\prime}$ ).

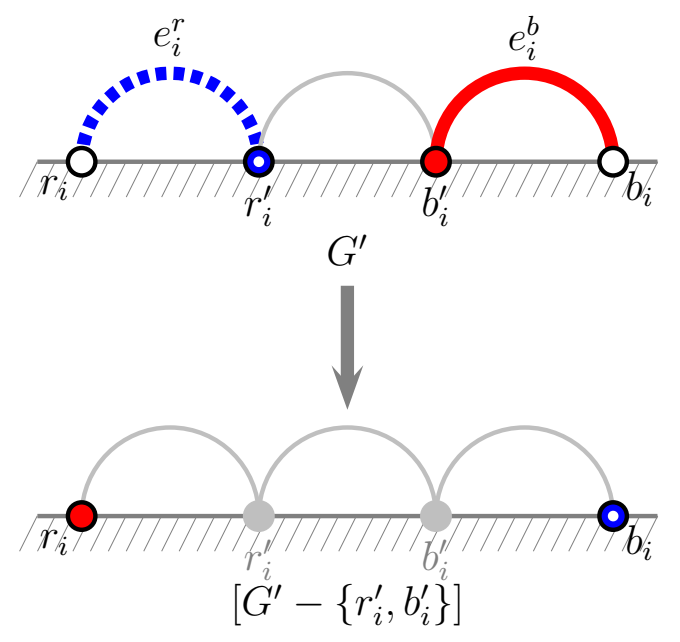

simply remove them together with $\left\{r_{i}^{\prime}, b_{i}^{\prime}\right\}$ from the corresponding subgraph (blue or red, respectively) of $G^{\prime}$, thus obtaining a superposition of matchings $\mu^{\prime \prime} \cup \nu^{\prime \prime}$ in the bicoloured graph $\left[G^{\prime}-\left\{r_{i}^{\prime}, b_{i}^{\prime}\right\}\right]_{\mathrm{b}^{\prime \prime} \mid \mathbf{r}^{\prime \prime}}$ with

- $\mathbf{b}^{\prime \prime}:=\mathbf{b}^{\prime} \cup\left\{b_{i}\right\} \backslash\left\{r_{i}^{\prime}\right\}$ and $\mathbf{r}^{\prime \prime}:=\mathrm{r}^{\prime} \cup\left\{r_{i}\right\} \backslash\left\{b_{i}^{\prime}\right\}$, if $r_{i}^{\prime} \in \mathbf{b}^{\prime}$ (as in Figure 14),

- $\mathbf{b}^{\prime \prime}:=\mathbf{b}^{\prime} \cup\left\{r_{i}\right\} \backslash\left\{b_{i}^{\prime}\right\}$ and $\mathrm{r}^{\prime \prime}:=\mathrm{r}^{\prime} \cup\left\{b_{i}\right\} \backslash\left\{r_{i}^{\prime}\right\}$, if $r_{i}^{\prime} \in \mathrm{r}^{\prime}$.

Observe that $\left[G^{\prime}-\left\{r_{i}^{\prime}, b_{i}^{\prime}\right\}\right]$ "locally looks like" the original graph $G$. For later use, note that

$$
\omega\left(\mu^{\prime} \cup \nu^{\prime}\right)=\omega\left(e_{i}\right) \times \omega\left(\mu^{\prime \prime} \cup \nu^{\prime \prime}\right)
$$

(there is no sign-change here, since the edges $e_{i}^{r}$ and $e_{i}^{b}$ in $G^{\prime}$ can never be involved in any crossing).

If $r_{i}^{\prime}$ and $b_{i}^{\prime}$ are of the same colour (say red), then for any superposition of matchings $\mu^{\prime} \cup \nu^{\prime}$ in $B^{\prime}$

- the blue subgraph $\left[G^{\prime}-\mathrm{r}^{\prime}\right]$ (which contains the blue matching $\nu^{\prime}$ ) "locally looks like" the original graph $G$ with edge $e_{i}$ removed (since this is "locally equivalent" to removing the two red vertices $r_{i}^{\prime}$ and $b_{i}^{\prime}$, see the lower left picture in Figure 15), 
Figure 15: Illustration of the construction preceding Lemma 7: If $r_{i}^{\prime}$ and $b_{i}^{\prime}$ are of the same colour (say red), then the construction shown in Figure 13 can be reversed in the "red subgraph" (see the right lower picture, which "locally" looks like the original graph $G$ ). This operation introduces a sign factor according to the considerations preceding (30). In the "blue subgraph" the vertices $r_{i}^{\prime}$ and $b_{i}^{\prime}$ simply are missing (see the left lower picture, which "locally" looks like the subgraph $\left.\left[G-e_{i}\right]\right)$.

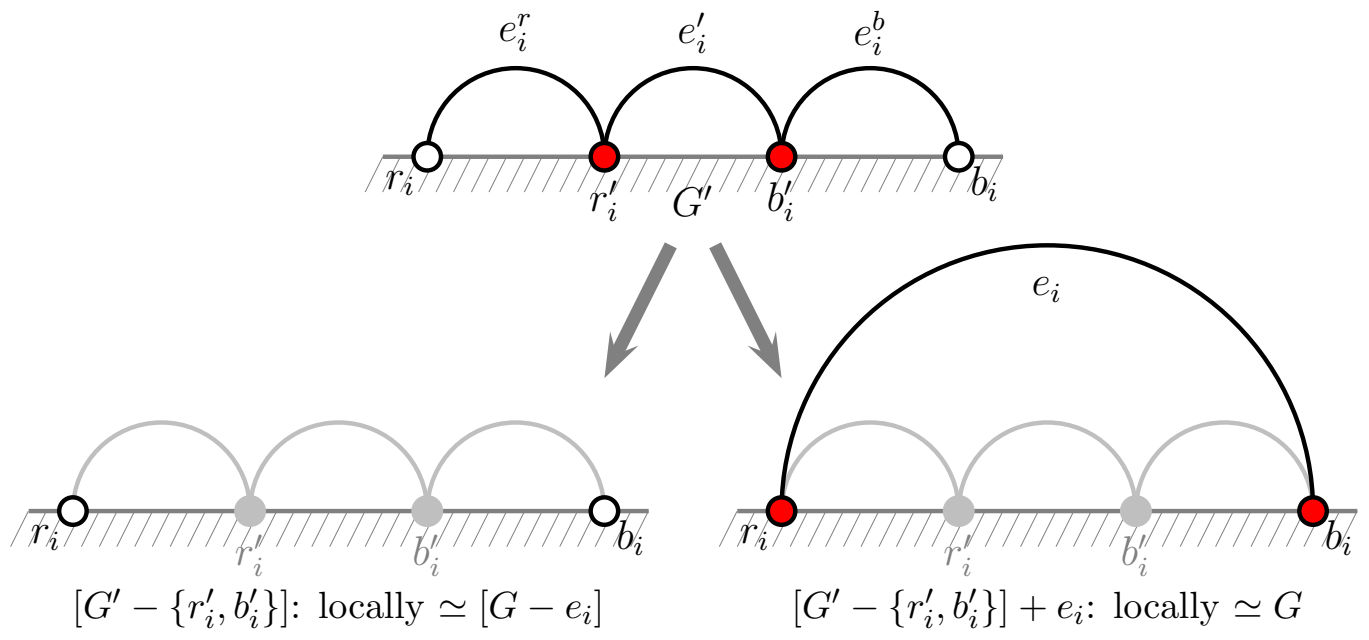

- and in the red subgraph $\left[G^{\prime}-\mathbf{b}^{\prime}\right]$ (which contains the red matching $\mu^{\prime}$ ) we may rereplace the path of length $3\left(r_{i}, r_{i}^{\prime}, b_{i}^{\prime}, b_{i}\right)$ in $G^{\prime}$ by the "original" edge $e_{i}=\left\{r_{i}, b_{i}\right\}$ in $G$ : This operation changes the corresponding Pfaffian only by a sign-factor according to the considerations preceding (30), and the resulting red subgraph "locally looks like" the original graph $G$ (see the lower right picture in Figure 15).

Applying these simple considerations to all pairs $\left(r_{i}^{\prime}, b_{i}^{\prime}\right), i=1, \ldots, k$ in $G^{\prime}$, we see that superpositions of matchings in $B^{\prime}=G_{\mathrm{b}^{\prime} \mid \mathrm{r}^{\prime}}^{\prime}$ are in bijection with superpositions of matchings in a certain bicoloured graph derived from $G$, which we now describe:

Define the sets of blue/red vertices in $G$, where the "primed partners with the same subscript" in $G^{\prime}$ are of different colours (see Figure 14):

$$
\begin{aligned}
\mathbf{b}^{\prime \prime} & :=\left\{r_{i}: r_{i}^{\prime} \in \mathrm{r}^{\prime} b_{i}^{\prime} \in \mathbf{b}^{\prime}\right\} \cup\left\{b_{i}: b_{i}^{\prime} \in \mathbf{r}^{\prime} r_{i}^{\prime} \in \mathbf{b}^{\prime}\right\}, \\
\mathbf{r}^{\prime \prime} & :=\left\{b_{i}: r_{i}^{\prime} \in \mathrm{r}^{\prime} b_{i}^{\prime} \in \mathbf{b}^{\prime}\right\} \cup\left\{r_{i}: b_{i}^{\prime} \in \mathbf{r}^{\prime} r_{i}^{\prime} \in \mathbf{b}^{\prime}\right\} .
\end{aligned}
$$

Define the sets of blue/red edges in $G$, whose end-vertices are "primed partners with the same subscript" in $G^{\prime}$ and of the same colour (see Figure 15):

$$
\begin{aligned}
\mathrm{r}_{e} & :=\left\{e_{i}: b_{i}^{\prime} \in \mathrm{r}^{\prime} r_{i}^{\prime} \in \mathrm{r}^{\prime}\right\}, \\
\mathbf{b}_{e} & :=\left\{e_{i}: b_{i}^{\prime} \in \mathbf{b}^{\prime} r_{i}^{\prime} \in \mathbf{b}^{\prime}\right\} .
\end{aligned}
$$


Finally, define the set of vertices incident with edges from $\mathbf{r}_{e} \cup \mathbf{b}_{e}$ :

$$
Z:=\left\{b_{i}, r_{i}: b_{i} \text { and } r_{i} \text { are of the same colour }\right\}=\left(\bigcup_{e \in \mathbf{r}_{e}} e\right) \cup\left(\bigcup_{e \in \mathbf{b}_{e}} e\right) .
$$

For an arbitrary graph $H$ and subsets $V^{\prime} \subseteq \mathrm{V}(H)$ and $E^{\prime} \subseteq \mathrm{E}(H)$ introduce the notation

$$
\left[H-V^{\prime}-E^{\prime}\right]
$$

for the graph $\left[H-V^{\prime}\right]$ with all edges in $E^{\prime}$ removed. Then the above reasoning amounts to the following Pfaffian identity:

$$
\begin{aligned}
& \operatorname{Pf}\left(\left[G^{\prime}-\mathbf{b}^{\prime}\right]\right) \cdot \operatorname{Pf}\left(\left[G^{\prime}-\mathrm{r}^{\prime}\right]\right)= \\
& \quad(-1)^{|Z|+\Sigma(Z \subseteq V)}\left(\prod_{e_{i} \notin\left(\mathbf{b}_{e} \cup \mathrm{r}_{e}\right)} \omega\left(e_{i}\right)\right) \cdot \operatorname{Pf}\left(\left[G-\mathbf{b}^{\prime \prime}-\mathbf{b}_{e}\right]\right) \cdot \operatorname{Pf}\left(\left[G-\mathrm{r}^{\prime \prime}-\mathbf{r}_{e}\right]\right) .
\end{aligned}
$$

These considerations lead to the following assertion:

Lemma 7. Assume the situation of Lemma 5 with the additional condition that there is no vertex between $r_{i}$ and $b_{i}$ in the ordered set of vertices $\mathrm{V}(G)\left(G=K_{V}\right)$. For an arbitrary subset $I \subseteq\{1, \ldots, k\}$ denote by $\bar{I}$ the subset $\{1, \ldots, k\} \backslash I$ and introduce the following "template notation":

$$
\mathbf{x}_{I}:=\left\{x_{i}: i \in I\right\},
$$

where $x$ may be any symbol from the set $\left\{r, r^{\prime}, b, b^{\prime}, e, e^{\prime}\right\}$.

Then we have for all fixed subsets $B \subseteq\{1, \ldots, k\}$ :

$$
\begin{gathered}
\sum_{R \subseteq\{1, \ldots, k\}}\left(\prod_{e_{i} \in \mathbf{e}_{\bar{R}}} \omega\left(e_{i}\right)\right) \cdot \operatorname{Pf}\left(\left[G-\mathbf{r}_{\bar{R}}\right]\right) \cdot \operatorname{Pf}\left(\left[G-\mathbf{b}_{\bar{R}}-\mathbf{e}_{R}\right]\right)= \\
\sum_{R \subseteq\{1, \ldots, k\}}\left(\prod_{e_{i} \in\left(\mathbf{e}_{\overline{R \triangle B}}\right)} \omega\left(e_{i}\right)\right) \times \\
\operatorname{Pf}\left(\left[G-\left(\mathbf{r}_{\bar{B} \cap \bar{R}} \cup \mathbf{b}_{R \cap B}\right)-\mathbf{e}_{B \backslash R}\right]\right) \cdot \operatorname{Pf}\left(\left[G-\left(\mathbf{b}_{\bar{R} \cap \bar{B}} \cup \mathbf{r}_{B \cap R}\right)-\mathbf{e}_{R \backslash B}\right]\right)
\end{gathered}
$$

This identity is of the involution-type.

Proof. Consider the graph $G^{\prime}$ as defined in the considerations preceding Lemma 7. It is obvious that every bicoloured path in $G$ connecting two vertices $b_{i}$ and $b_{j}$ (or $r_{i}$ and $r_{j}$ ) corresponds bijectively to a bicoloured path in $G^{\prime}$ connecting the vertices $b_{i}^{\prime}$ and $b_{j}^{\prime}$ (or $r_{i}^{\prime}$ and $r_{j}^{\prime}$ ), which thus also has weight zero. Hence the weight function of $G^{\prime}$ is also planar, 
and we may apply (28) from Lemma 5 to $G^{\prime}$ : The rest of this proof consists of a mere "translation".

Consider the generic summand

$$
\operatorname{Pf}(((\mathbf{R} \cup \mathbf{w}) \cup V) \backslash X) \cdot \operatorname{Pf}(((\mathbf{B} \cup \mathbf{w}) \backslash V) \cup X)
$$

involved in (28): At first sight, this is the summand only for the right-hand side of (28), but note that specializing $X=\emptyset$ gives the summand of the left-hand side.

We rewrite this summand for $G^{\prime}$ with $\mathbf{B}^{\prime}=\left\{b_{1}^{\prime}, \ldots, b_{k}^{\prime}\right\}$ and $\mathbf{R}^{\prime}=\left\{r_{1}^{\prime}, \ldots, r_{k}^{\prime}\right\}$ as follows: Let $R, B \subseteq\{1, \ldots, k\}$ such that $V=\mathbf{b}^{\prime}{ }_{R} \subseteq \mathbf{B}^{\prime}$ and $X=\mathbf{r}_{B}^{\prime} \subseteq \mathbf{R}^{\prime}$. Then we may write

$$
\operatorname{Pf}\left(\left(\left(\mathbf{R}^{\prime} \cup \mathbf{w}\right) \cup V\right) \backslash X\right) \cdot \operatorname{Pf}\left(\left(\left(\mathbf{B}^{\prime} \cup \mathbf{w}\right) \backslash V\right) \cup X\right)=\operatorname{Pf}\left(\left[G^{\prime}-\mathbf{b}^{\prime}\right]\right) \operatorname{Pf}\left(\left[G^{\prime}-\mathbf{r}^{\prime}\right]\right),
$$

where $\mathbf{b}^{\prime}=\mathbf{r}_{B}^{\prime} \cup \mathbf{b}_{\bar{R}}^{\prime}$ and $\mathbf{r}^{\prime}=\mathbf{b}_{R}^{\prime} \cup \mathbf{r}_{\bar{B}}^{\prime}$.

Then the definitions of $\mathbf{b}^{\prime \prime}$ and $\mathbf{r}^{\prime \prime}$ (according to (32)) and of $\mathbf{b}_{e}$ and $\mathbf{r}_{e}$ (according to (33)) can be written as:

$$
\begin{aligned}
\mathbf{b}^{\prime \prime} & =\mathbf{r}_{\bar{B} \cap \bar{R}} \cup \mathbf{b}_{R \cap B}, \\
\mathbf{r}^{\prime \prime} & =\mathbf{b}_{\bar{R} \cap \bar{B}} \cup \mathbf{r}_{B \cap R}, \\
\mathbf{b}_{e} & =\mathbf{e}_{B \backslash R}, \\
\mathbf{r}_{e} & =\mathbf{e}_{R \backslash B} .
\end{aligned}
$$

So altogether, (34) translates to:

$$
\begin{gathered}
\operatorname{Pf}\left(\left[G^{\prime}-\mathbf{b}^{\prime}\right]\right) \operatorname{Pf}\left(\left[G^{\prime}-\mathbf{r}^{\prime}\right]\right)=\left(\prod_{e_{i} \in\left(\mathbf{e}_{\overline{R \triangle B}}\right)} \omega\left(e_{i}\right)\right) \times \\
\operatorname{Pf}\left(\left[G-\left(\mathbf{r}_{\bar{B} \cap \bar{R}} \cup \mathbf{b}_{R \cap B}\right)-\mathbf{e}_{B \backslash R}\right]\right) \cdot \operatorname{Pf}\left(\left[G-\left(\mathbf{b}_{\bar{R} \cap \bar{B}} \cup \mathbf{r}_{B \cap R}\right)-\mathbf{e}_{R \backslash B}\right]\right) .
\end{gathered}
$$

(Note that there is no sign-change here, since there are no vertices between $r_{i}$ and $b_{i}$ by assumption.)

Putting together (37) and (38) gives

$$
\begin{array}{r}
\operatorname{Pf}\left(\left(\left(\mathbf{R}^{\prime} \cup \mathbf{w}\right) \cup V\right) \backslash X\right) \cdot \operatorname{Pf}\left(\left(\left(\mathbf{B}^{\prime} \cup \mathbf{w}\right) \backslash V\right) \cup X\right)=\left(\prod_{e_{i} \in\left(\mathbf{e}_{\overline{R \triangle B}}\right)} \omega\left(e_{i}\right)\right) \times \\
\operatorname{Pf}\left(\left[G-\left(\mathbf{r}_{\bar{B} \cap \bar{R}} \cup \mathbf{b}_{R \cap B}\right)-\mathbf{e}_{B \backslash R}\right]\right) \cdot \operatorname{Pf}\left(\left[G-\left(\mathbf{b}_{\bar{R} \cap \bar{B}} \cup \mathbf{r}_{B \cap R}\right)-\mathbf{e}_{R \backslash B}\right]\right),
\end{array}
$$

and inserting this into (28) (set $B=\emptyset$ for the left-hand side of (28)) gives the translation to $(35)$. 
This yields immediately the following generalization of a result by Yan, Yeh and Zhang [23, Theorem 3.2] (here, we write the summands for $\bar{R}$ instead of $R$ when summing over all $R \subseteq\{1, \ldots, k\}$, to make the equivalence with [23, equation (8)] more transparent):

Corollary 8. Let $G$ be a planar graph with $k$ independent edges $e_{i}=\left\{a_{i}, b_{i}\right\}, i=1, \ldots, k$, in the boundary of some face $f$ of $G$, such that the vertices $a_{1}, b_{1}, \ldots, a_{k}, b_{k}$ appear in that cyclic order in $f$.

Then we have for all fixed subsets $B \subseteq\{1, \ldots, k\}$ :

$$
\begin{aligned}
\sum_{R \subseteq\{1, \ldots, k\}}\left(\prod_{e_{i} \in \mathbf{e}_{R}} \omega\left(e_{i}\right)\right) \cdot M_{\left[G-\mathbf{r}_{R}\right]} \cdot M_{\left[G-\mathbf{b}_{R}-\mathbf{e}_{\bar{R}}\right]}= \\
\quad \sum_{R \subseteq\{1, \ldots, k\}}\left(\prod_{e_{i} \in\left(\mathbf{e}_{R \triangle B}\right)} \omega\left(e_{i}\right)\right) \cdot M_{\left[G-\left(\mathbf{r}_{\bar{B} \cap R} \cup \mathbf{b}_{\bar{R} \cap B}\right)-\mathbf{e}_{B \backslash \bar{R}}\right]} \cdot M_{\left[G-\left(\mathbf{b}_{R \cap \bar{B}} \cup \mathbf{r}_{B \cap \bar{R}}\right)-\mathbf{e}_{\bar{R} \backslash B}\right]}
\end{aligned}
$$

Proof. The statement follows from (35) by the Kasteleyn-Percus method.

\section{References}

[1] A. Cayley. Sur les déterminants gauches. Journal für die reine und angewandte Mathematik, 38:93-96, 1849.

[2] A. Cayley. On the theory of permutants. Cambridge and Dublin Mathematical Journal, 7:40-51, 1852.

[3] M. Ciucu. Enumeration of perfect matchings in graphs with reflective symmetry. $J$. Combin. Theory Ser. 1, 77:67-97, 1997.

[4] R. Diestel. Graph Theory. Springer, 2nd edition, 2000.

[5] A. Dress and W. Wenzel. A simple proof of an identity concerning Pfaffians of skew symmetric matrices. Adv. Math., 112:120-134, 1995.

[6] A. M. Hamel. Pfaffian identities: A combinatorial approach. Journal Combin. Theory A, 94:205-217, 2001.

[7] P.W. Kasteleyn. Dimer statistics and phase transitions. J. Mathematical Phys., 4(2):287-293, 1963.

[8] P.W. Kasteleyn. Graph theory and crystal physics. In F. Harary, editor, Graph Theory and Theoretical Physics, chapter 2, pages 43-110. Academic Press, 1967.

[9] D.E. Knuth. Overlapping pfaffians. Electron. J. Combin., 3(2):\#R5, 1996.

[10] C. Krattenhaler. private communication. July 6, 1995.

[11] E. H. Kuo. Graphical condensation generalizations involving Pfaffians and determinants. math:CO/0605154. 
[12] E. H. Kuo. Applications of graphical condensation for enumerating matchings and tilings. Theoret. Comput. Sci., 319:29-57, 2004.

[13] F. Mertens. Über die Determinanten, deren correspondirende Elemente $a_{p q}$ und $a_{q p}$ entgegengesetzt gleich sind. Journal für die reine und angewandte Mathematik, $82: 207-211,1877$.

[14] Yasuhiro Ohta. Pfaffian [text in Japanese]. RIMS Kôkyûroku, 822(197-205), 1993.

[15] J. K. Percus. One more technique for the dimer problem. J. Mathematical Phys., 10(10):1881-1884, 1969.

[16] J. Propp. Generalized domino-shuffling. Theoret. Comput. Sci., 303:267-301, 2003.

[17] H. Srinivasan. Decomposition formulas for Pfaffians. J. Algebra, 163(2):312-334, 1994.

[18] John R. Stembridge. Nonintersecting paths, pfaffians and plane partitions. Adv. Math., 83:96-131, 1990.

[19] H.W. Lloyd Tanner. A theorem relating to Pfaffians. Messenger of Mathematics, $8: 56-59,1878$.

[20] W. Veltmann. Beiträge zur Theorie der Determinanten. Zeitschrift für Mathematik und Physik, 16:516-525, 1871.

[21] W. Wenzel. Pfaffian forms and $\Delta$-matroids. Discrete Mathematics, 115:253-266, 1993.

[22] W. Yan and F. Zhang. Graphical condensation for enumerating perfect matchings. Journal Combin. Theory A, 110:113-125, 2005.

[23] W.G. Yan, Y.N. Yeh, and F.J. Zhang. Graphical condensation of plane graphs: a combinatorial approach. Theoret. Comput. Sci., 349:452-461, 2005. 